\title{
Syntaxin 5 determines Weibel-Palade body size and von Willebrand factor secretion by controlling Golgi architecture
}

Marije Kat, ${ }^{1 \star}$ Ellie Karampini, ${ }^{1,2^{\star}}$ Arie J. Hoogendijk, ${ }^{1}$ Petra E. Bürgisser, ${ }^{3}$ Aat A. Mulder, ${ }^{4}$ Floris P.J. van Alphen, ${ }^{1}$ Jenny Olins, ${ }^{1}$ Dirk Geerts, ${ }^{5}$ Maartje van den Biggelaar, ${ }^{1}$ Coert Margadant, ${ }^{6}$ Jan Voorberg ${ }^{1,7}$ and Ruben Bierings ${ }^{3}$

\footnotetext{
'Molecular Hematology, Sanquin Research and Landsteiner Laboratory, Amsterdam University Medical Center, University of Amsterdam, Amsterdam, the Netherlands; ${ }^{2}$ Vascular Biology, Royal College of Surgeons, Dublin, Ireland; ${ }^{3}$ Hematology, Erasmus University Medical Center, Rotterdam, the Netherlands; ${ }^{4}$ Molecular Cell Biology, Leiden University Medical Center, Leiden, the Netherlands; ${ }^{5}$ Medical Biology, Amsterdam University Medical Center, location AMC, University of Amsterdam, Amsterdam, the Netherlands; ${ }^{6}$ Angiogenesis Laboratory, Cancer Center Amsterdam, Amsterdam University Medical Center, location VUmc, Amsterdam, the Netherlands and ${ }^{7}$ Experimental Vascular Medicine, Amsterdam University Medical Center, University of Amsterdam, Amsterdam, the Netherlands.
}

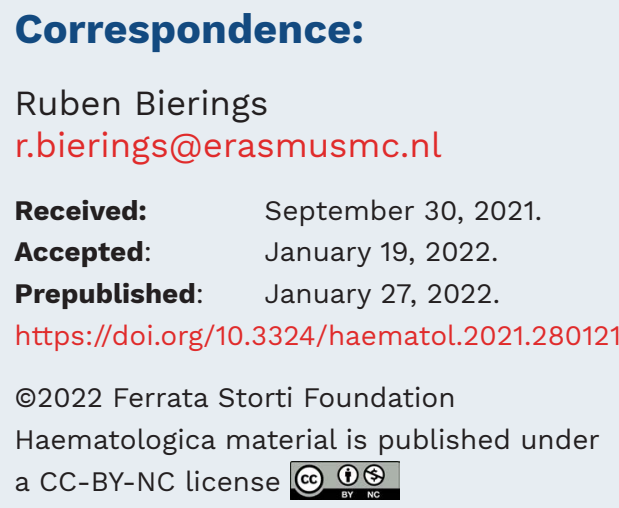

*MK and EK contributed equally as co-first authors.

\begin{abstract}
von Willebrand factor (VWF) is a multimeric hemostatic protein primarily synthesized in endothelial cells. VWF is stored in endothelial storage organelles, the Weibel-Palade bodies (WPB), whose biogenesis strongly depends on VWF anterograde trafficking and Golgi architecture. Elongated WPB morphology is correlated to longer VWF strings with better adhesive properties. We previously identified the SNARE SEC22B, which is involved in anterograde endoplasmic reticulum-to-Golgi transport, as a novel regulator of WPB elongation. To elucidate novel determinants of WPB morphology we explored endothelial SEC22B interaction partners in a mass spectrometry-based approach, identifying the Golgi SNARE Syntaxin 5 (STX5). We established STX5 knockdown in endothelial cells using shRNA-dependent silencing and analyzed WPB and Golgi morphology, using confocal and electron microscopy. STX5-depleted endothelial cells exhibited extensive Golgi fragmentation and decreased WPB length, which was associated with reduced intracellular VWF levels, and impaired stimulated VWF secretion. However, the secretion-incompetent organelles in shSTX5 cells maintained WPB markers such as Angiopoietin 2, P-selectin, Rab27A, and CD63. In brief, we identified SNARE protein STX5 as a novel regulator of WPB biogenesis.
\end{abstract}

\section{Introduction}

von Willebrand factor (VWF) is a hemostatic glycoprotein that is primarily synthesized in endothelial cells (EC) and acts as a factor VIII chaperone as well as an adhesive grid for thrombus formation.' Decreased VWF plasma levels or mutations in the VWF gene can cause von Willebrand disease (VWD), the most common bleeding disorder. ${ }^{2}$ VWF undergoes a multistep maturation process that involves dimerization in the endoplasmic reticulum (ER), followed by multimerization and proteolytic processing in the Golgi.' Smaller VWF multimers are continuously secreted (primarily) at the basolateral surface via the constitutive pathway, while larger VWF multimers are condensed into storage organelles emerging from the trans-Golgi network (TGN): the Weibel-Palade bodies (WPB). ${ }^{3}$

WPB biogenesis is tightly linked to VWF synthesis, which is highlighted by the absence of WPB in VWF knockout EC, and their de novo formation in non-EC by ectopic VWF expression. ${ }^{4-6}$ Besides VWF, WPB contain inflammatory and angiogenic proteins, and recruit essential transport and exocytotic machinery. ${ }^{7,8}$ WPB exocytosis occurs via basal (continuous) or regulated (stimulus-induced) secretion pathways, both predominantly targeting the apical surface facing the blood vessel lumen. ${ }^{3}$ WPB play an important role during primary hemostasis as their release ensures the immediate delivery of VWF (and other molecules) in the vessel lumen in response to injury, whereupon VWF 
tubules unfurl into long VWF strings on the apical surface, which subsequently become decorated by platelets. $^{8}$

WPB have a distinct, elongated morphology: the cigarshaped structure is composed of densely packed helical tubules of VWF multimers running along the length of the organelle enwrapped by a tightly fitted endomembrane..$^{9,10}$ Both the length and adhesive properties of VWF strings correlated with WPB length; shorter WPB generate shorter VWF strings, with lower adhesive capacity for platelets and plasma VWF.1112 However, what drives their distinct morphology is still largely unknown. The range in WPB size was defined by the VWF quanta model, which describes how during biogenesis VWF nanoclusters of a discrete length (i.e., quanta) are co-packaged in variable numbers at the Golgi, ultimately determining the length of the WPB..$^{13}$ Although WPB length is known to be determined by Golgi ribbon architecture as well as by levels of VWF synthesis, ${ }^{13,14}$ only recently has the control of VWF progression through the early secretory pathway been appreciated as a determinant of WPB length..$^{15-17}$

In anterograde transport vesicles bud off at ER exit sites, containing specific cargo en route to the Golgi. During this process soluble $\mathrm{N}$-ethylmaleimide-sensitive fusion protein attachment protein receptors (SNARE) are incorporated in the vesicle membrane (i.e., v-SNARE), which can form complexes with Golgi-associated target membrane SNARE (t-SNARE) to facilitate membrane fusion and cargo release in the Golgi lumen. ${ }^{18,19}$ One study showed that the ARF guanine-nucleotide exchange factor (GEF) Golgi Brefeldin A-resistant GEF 1 (GBF1) modulates vesicle fission at the ER and TGN, having an impact on WPB size by controlling anterograde VWF transport and WPB segregation from the TGN. ${ }^{15}$

Exocytic SNARE proteins play a key role in WPB exocytosis, ${ }^{20-24}$ and some have also been associated with VWF plasma levels and severity of VWD. ${ }^{25,26}$ The role of SNARE proteins in WPB biogenesis and VWF trafficking, however, remains elusive. We have recently characterized the $v$ SNARE SEC22B as a novel WPB size regulator through its role in anterograde VWF transport and support of elongated Golgi morphology. ${ }^{16}$ In the current study, we aimed at identifying novel determinants of VWF trafficking through mapping ER-Golgi fusion machinery in EC by elucidating the SEC22B interactome. The proteomic screen revealed a plethora of potential interactors, including the SNARE protein syntaxin 5 (STX5). Knockdown of STX5 in EC resulted in extensive fragmentation of Golgi architecture, VWF retention in the ER, and significantly shorter and fewer WPB. In addition, both intracellular VWF levels and regulated WPB exocytosis were significantly suppressed, highlighting STX5 as an essential component of the machinery driving WPB biogenesis and release.

\section{Methods}

\section{Mass spectrometry analysis}

Sample preparation, data acquisition and data analysis were performed as previously described. ${ }^{27}$ Detailed experimental procedures are described in the Online Supplementary Material.

\section{von Willebrand factor string assay}

VWF string assays were essentially performed as previously described using 75,000 shCTRL or shSTX5 transduced cells per channel in gelatin-coated six-channel IBIDI $\mu$-slides VI 0.4. ${ }^{28}$ Strings were visualized by supplementing perfusion mix with AF488-conjugated anti-VWF antibody (DAKO) at $2 \mu \mathrm{M}$ concentration. Further experimental details can be found in the Online Supplementary Material.

\section{Secretion assay}

EC were transduced and grown in six-well plates and cultured for 7 days prior to the experiment with regular replacement of medium. Basal VWF release was determined as unstimulated secretion over 48 h. For histamine-induced secretion cells were starved for $30 \mathrm{~min}$ in $\mathrm{M}-199$ with $1 \%$ bovine serum albumin and subsequently stimulated with $100 \mu \mathrm{M}$ histamine (Sigma-Aldrich; H7125) for $1 \mathrm{~h}$. Lysates were obtained in lysis buffer (phosphatebuffered saline, $1 \%$ Triton $X-100$ ) supplemented with Halt protease and a phosphatase inhibitor cocktail. VWF levels were determined by enzyme-linked immunosorbent assay as described previously. ${ }^{21}$

Antibodies and DNA constructs are listed in Online Supplementary Tables $S 1$ and S2, respectively. Additional methods can be found in the Online Supplementary Material.

\section{Results}

The SEC22B interactome in endothelial cells contains SNARE proteins involved in anterograde, retrograde and intra-Golgi protein trafficking.

To map the composition of the ER-to-Golgi SNARE networks that control VWF trafficking and WPB biogenesis, we employed unbiased affinity purification-mass spectrometry in EC using mEGFP-tagged SEC22B as bait. A total of 841 proteins were significantly enriched in mEGFP-SEC22B compared to the mEGFP control (Figure 1A, B, Online Supplementary Table S3). Gene ontology enrichment analysis revealed that the most prominent complexes within the cellular components ontology were 'membrane protein' 'inner mitochondrial membrane protein', and 'SNARE' (Figure 1C). Furthermore, SEC22B and its potential interacting partners collaborated in 235 en- 
riched biological processes, including anterograde and retrograde ER-Golgi trafficking, intra-Golgi trafficking as well as Golgi organization (Online Supplementary Table S4).

To visualize protein interactions a STRING network was generated based on high confidence interactions, showing two major clusters representing proteins that are part of the inner MT membrane protein complex or a SNARE complex, with SEC22B as part of the latter (Figure 1D). Among the significant hits there were many SNARE, including STX5 and GOSR2 (Golgi SNAP Receptor Complex Member 2), which are part of the anterograde SNARE complex, and STX18, which facilitates retrograde trafficking. ${ }^{29}$ Another protein complex represented in the STRING analysis comprises components of the vesicle-tethering NBAS/RINT1/ZW10 (NRZ) complex (i.e., NAPA, NBAS, SCFD1, SCFD2, ZW10 and C19orf25), which regulates SNARE complex formation of incoming vesicles on the ER membrane. ${ }^{30}$ In addition, members of the conserved oligomeric Golgi (COG) complex were identified (i.e., COG16 and COG8), suggesting a link between SEC22B and the
intra-Golgi vesicle membrane tethering complex. ${ }^{31,32} \mathrm{~A}$ last notable hit in the SEC22B interactome screen is GBF1, which was previously implicated in ER-Golgi transport of VWF.15

The SEC22B interactome analysis uncovered a large protein network containing both known and novel candidates of protein trafficking in the endothelial biosynthetic pathway. Based on interaction score and the number of edges directed to SEC22B in the STRING analysis, we selected one of the most prominent hits within the SNARE cluster, STX5, for further follow-up. STX5 takes part in both anterograde ER-to-Golgi trafficking and in retrograde intraGolgi trafficking. ${ }^{29}$ We validated the interaction between STX5 and GFP-SEC22B by immunoblotting for co-precipitated endogenous STX5 (Figure 1E), which is expressed as long ( $40 \mathrm{kDa}$; STX5L) and short ( $34 \mathrm{kDa}$; STX5S) isoforms, resulting from an alternative start codon. ${ }^{33}$ Furthermore, immunofluorescent STX5 staining in human umbilical vein EC showed a perinuclear localization, overlapping with the trans-Golgi network marker TGN46 (Online Supplementary Figure S1A). SEC22B also concentrated
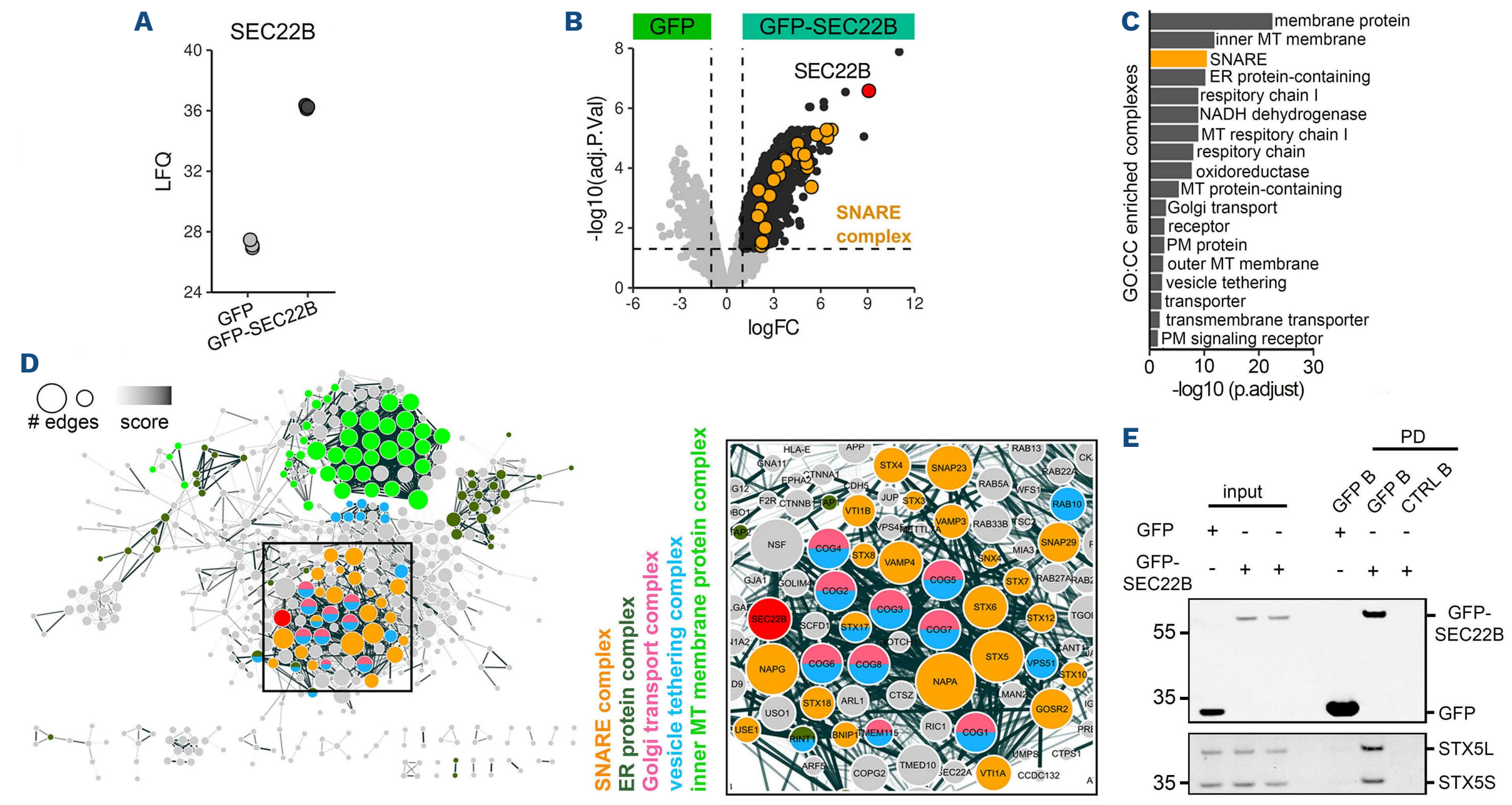

Figure 1. STX5 is part of the SEC22B interactome in endothelial cells. (A) Label-free quantification (LFQ) of SEC22B protein levels in pulldown samples determined in triplicate by mass spectrometry analysis. (B) Volcano plot of significantly enriched proteins in the mEGFP and mEGFP-SEC22B pulldown samples. Red dot represents SEC22B used as bait. Orange dots represent SNARE complex proteins based on GO:0031201 annotation. Dotted lines indicate significance thresholds ( $P$.adjust <0.05 and $|L F C|>1)$. (C) Bar plot of enriched GO:CC protein complexes. (D) STRING-DB analysis of the enriched proteins in mEGFP-SEC22B showing high confidence interactors (combined STRING-DB scores $>0.9$ ). Colors represent enriched complexes: SNARE complex (orange), endoplasmic reticulum (ER) protein-containing complex (dark green), Golgi transport complex (pink), Vesicle tethering complex (blue) and inner MT membrane protein complex (green). Red node indicates SEC22B. Disconnected nodes are not shown. The panel below shows a zoom of the area with high SNARE complex protein density. (E) Western blot analysis of the short (S) and long (L) isoforms of STX5 in input and pulldown (PD) samples from human umbilical vein endothelial cells expressing mEGFP or mEGFP-SEC22B. B: beads. 
in the Golgi area, but was additionally observed in small punctae localized in a wider area around the nucleus (Online Supplementary Figure S1B), most likely representing ER exit sites and trafficking vesicles. ${ }^{34}$ Collectively, these observations suggest that STX5 and SEC22B interact at the Golgi apparatus.

\section{STX5 and SEC22B depletion in endothelial cells induces unique and shared whole proteome alterations}

To study the role of STX5 in EC, we silenced its expression by stable expression of short hairpin (sh)RNA targeting STX5. We determined knockdown efficiency of five shRNA targeting STX5 in comparison to a non-targeting shRNA control (shCTRL). Two shRNA (shSTX5_59826 and shSTX5_59827) efficiently reduced expression of both STX5 isoforms (Online Supplementary Figure S2A, B).

To assess the impact of STX5 silencing in an unbiased manner, we explored differences on the proteomic level between shSTX5 and ShSEC22B compared to ShCTRL and untransduced cells, to determine the similarities and differences between silencing either of the two interacting partners in EC. Samples from the same condition clustered together in the principal component analysis, showing minimal variability between replicates (Figure 2A). After confirming knockdown of SEC22B and STX5 (Figure 2B, Online Supplementary Figure S3), we analyzed the sig- nificantly changed proteins (Online Supplementary Table S5). Analysis of proteomic alterations between the shSTX5 and shSEC22B conditions revealed 48 overlapping proteins, which included VWF and angiopoietin-2 (Ang-2), suggesting that STX5- and SEC22B-dependent intracellular trafficking regulates the transport of multiple WPB cargo proteins (Figure 2C). Moreover, SEC22B depletion rendered a total of 176 unique significantly changed proteins, whereas in shSTX5 cells only 59 proteins were altered.

To further examine these hits and assess protein co-regulation, we generated a co-expression heatmap based on Pearson correlations, which visualized four differentially regulated protein clusters (Figure 2D). These clusters revealed proteins that were mainly driven by STX5 (clusters 1 and 4) or shared between SEC22B and STX5 (cluster 2). Further inspection of cluster 3 highlighted the presence of WPB proteins, as illustrated by the decrease in intracellular VWF levels in both shSTX5 and ShSEC22B, and in addition a reduction in Ang-2 and multimerin 1 (MMRN1) (Figure 2E). Rab3D and VAMP3, which have previously been implicated in WPB formation and exocytosis, ${ }^{23,35}$ were only significantly downregulated in shSEC22B cells, but their expression levels appeared to be lower in shSTX5_59826 as well.

In summary, STX5 and SEC22B depletion causes overlap-
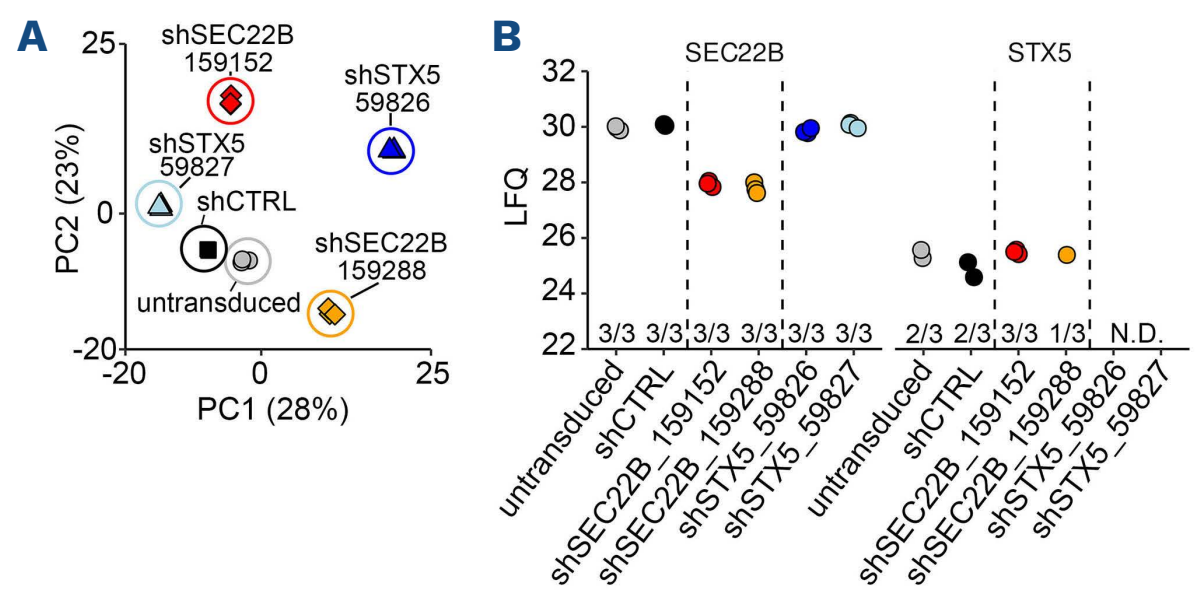

D

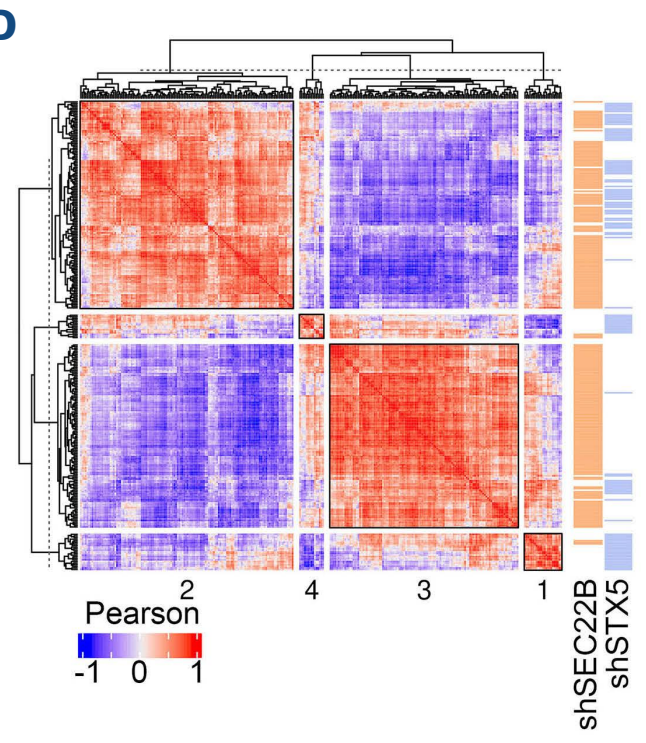

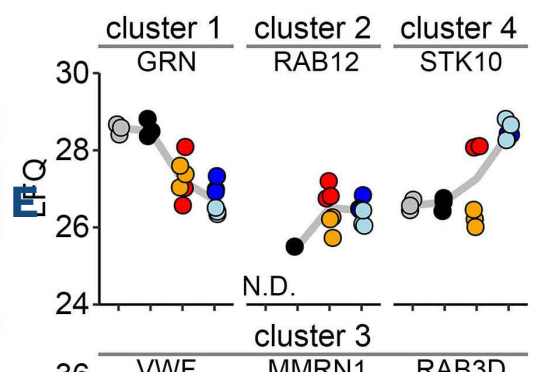

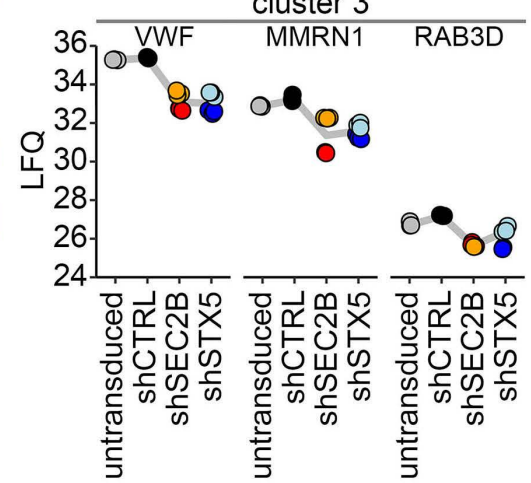

C

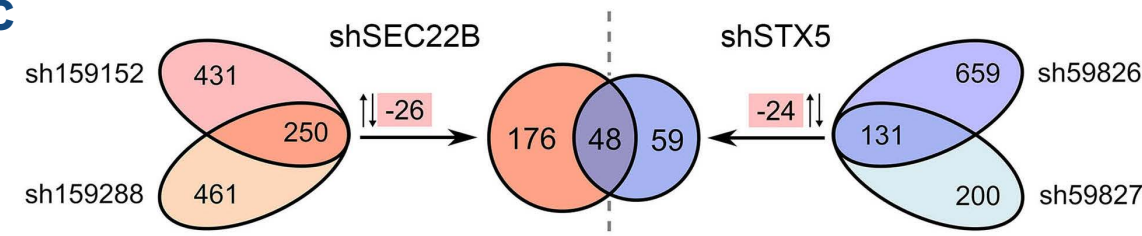

Figure 2. Whole proteome analysis reveals clusters of differentially regulated proteins in shSTX5 and shSEC22B endothelial cells. (A) Principal component (PC) analysis of analyzed samples in triplicate. (B) Label-free quantification (LFQ) plot showing mass spectrometry-based SEC22B and STX5 protein levels. (C) Venn diagrams showing overlap between individual shRNA and number of shared proteins between shSEC22B and shSTX5 within differentially regulated proteins compared to ShCTRL and untransduced conditions. Red boxes show the amount of shared proteins for which the direction of regulation was opposed (up vs. down). (D) Correlation heatmap showing Pearson coefficients of regulated proteins separated into four clusters. Row annotation indicates if a protein was regulated by SEC22B (orange), STX5 (blue) or both. (E) LFQ plots of cluster members. N.D.: not detected. 
ping proteomic changes, but STX5 knockdown also induces unique alterations in the proteome, highlighting the importance of STX5-mediated protein transport.

\section{STX5 silencing results in altered Weibel-Palade body length and loss of the Golgi architecture}

To address a potential role of STX5 in WPB biogenesis we investigated WPB and Golgi morphology in shSTX5 and shCTRL human umbilical vein EC. STX5 knockdown was validated by strongly reduced expression on western blot and (nearly) absent immunofluorescent staining upon STX5 knockdown (Figure 3A, B). Since shSTX5_59826 yields the most efficient and consistent knockdown, we selected this shRNA for further experiments. To confirm that the effects were specific for STX5 knockdown, key experiments were also performed with shSTX5_59827. VWF staining revealed that upon STX 5 silencing the characteristic elongated shape of WPB was lost, while the VWF that was present concentrated in spherical granules mostly found in the perinuclear area (Figure 3C). Assessment of immunogold-labeled VWF by electron microscopy confirmed that these VWF-positive structures were indeed short WPB (Online Supplementary Figure S4). Quantitative analysis of the size of VWF-positive structures revealed that WPB length was drastically decreased after depletion of STX5 (Figure 3D). We also observed a concomitant disintegration of the Golgi; while TGN46 staining showed a compact, continuous ribbon structure in shCTRL cells, shSTX5 cells showed TGN46 staining on dispersed, unlinked structures (Figure $3 \mathrm{C}$ ). Quantification revealed that in the shCTRL condition approximately $70 \%$ of the cells contained a continuous, compact Golgi, but the vast majority ( 90\%) of Golgi structures in shSTX5 cells appeared fragmented and dispersed (Figure 3E; examples of Golgi states shown below). Co-staining of TGN46 with the cis-Golgi marker GM130 revealed a similar dispersed phenotype for the cis-Golgi in shSTX5 cells, suggesting that the entire Golgi architecture is affected by STX5 de-
A
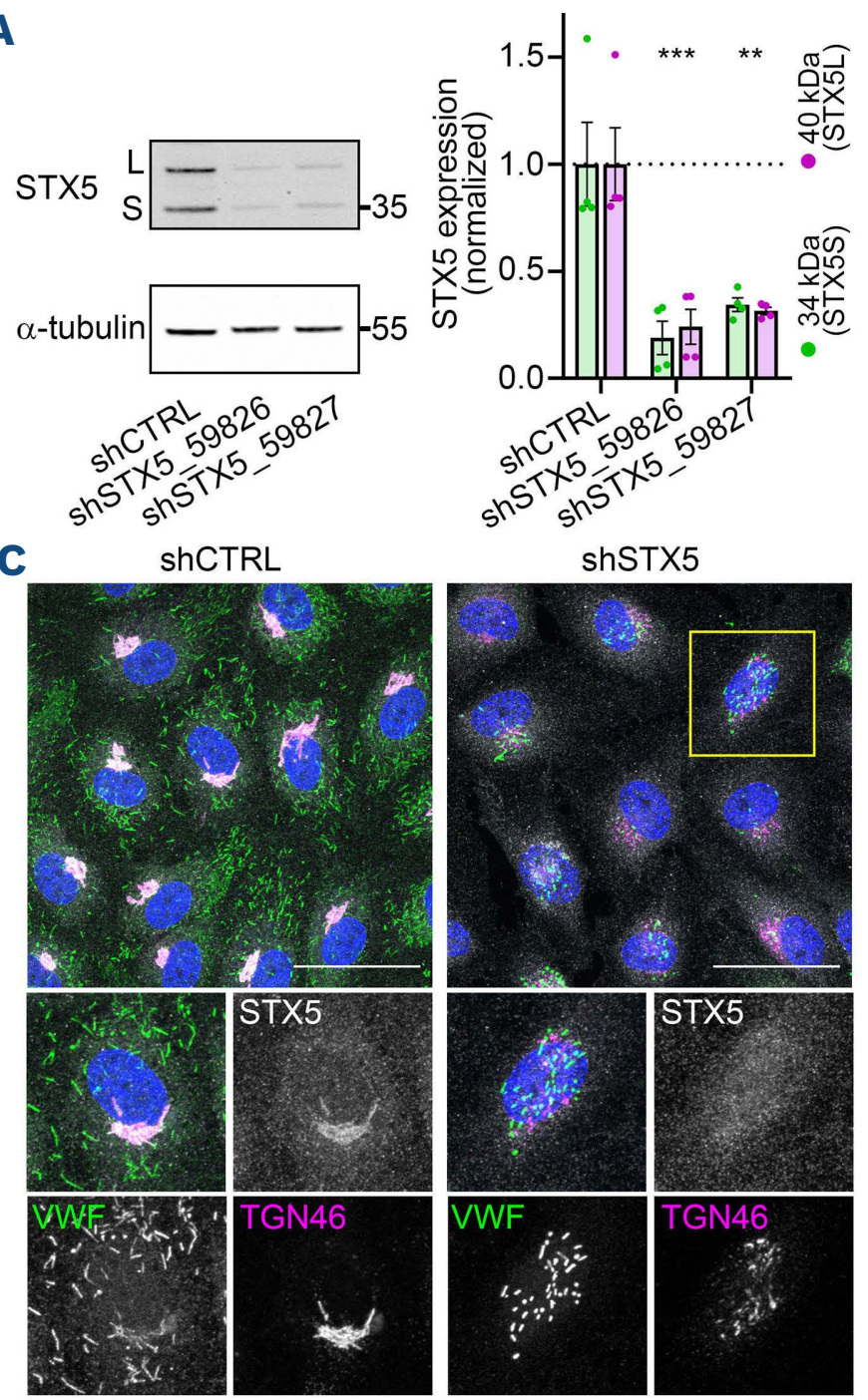

E

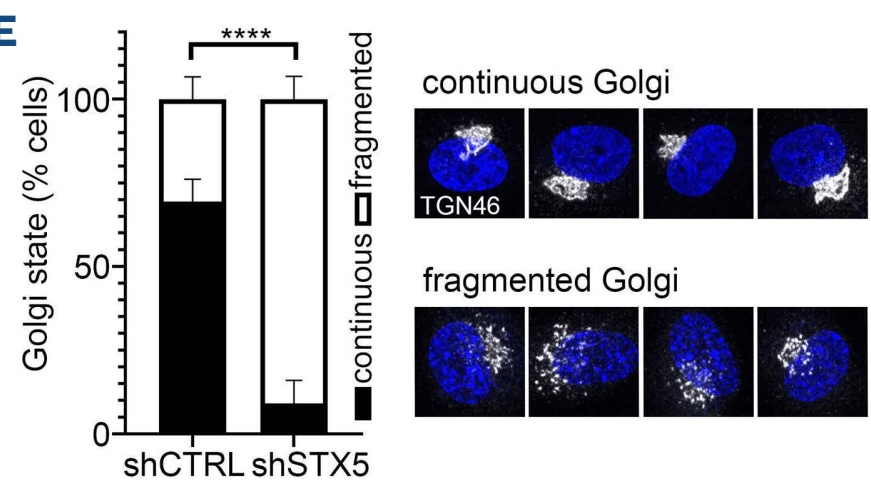

B
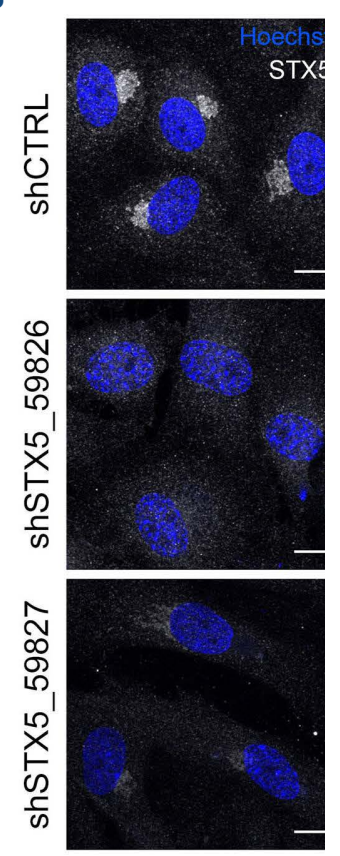

D
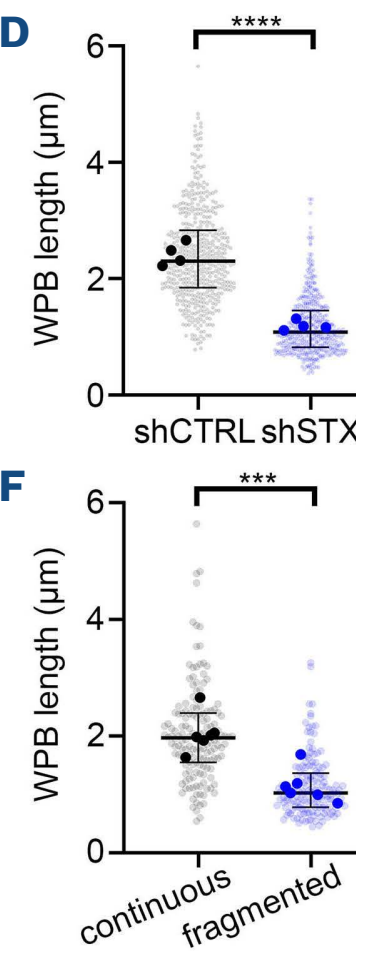

Figure 3. STX5 downregulation results in decreased Weibel-Palade body length and extensive Golgi fragmentation. (A) Western blot analysis of STX5 in ShCTRL and shSTX5 transduced human umbilical vein endothelial cells ( $\alpha$-tubulin as loading control) and quantification of STX5 knockdown efficiency (mean \pm standard error of the mean (SEM), $\mathrm{n}=4, t$ test, $* \star P<0.01, * * * P<0.001)$. Molecular weights of protein marker bands are indicated in $\mathrm{kDa}$. $\mathrm{L}$ and $\mathrm{S}$ indicate long and short STX5 isoforms, respectively. (B) Immunofluorescent staining of STX5 (gray) and nuclei (blue) in shCTRL and shSTX5 cells (scale bar: $10 \mu \mathrm{m})$. (C) Representative immunofluorescence microscopy images ( $n=7$ biological replicates) of staining for VWF (green), TGN46 (magenta), STX5 (gray), and nuclei (Hoechst; blue) in shCTRL and shSTX5 cells (scale bar: $40 \mu \mathrm{m}$ ). Boxed areas are magnified below (individual channels in gray scale). (D) Weibel-Palade body (WPB) length in shCTRL and shSTX5 cells measured in micrometers $(\mu \mathrm{m})$. Each transparent dot represents a WPB; the average length of each biological replicate is shown in filled color (median \pm interquartile range [IR], $\mathrm{n}=4, t$-test, $\star * * * P<0.0001)$. (E) The percentage of cells containing a continuous or fragmented Golgi in ShCTRL and shSTX5 cells (median \pm IR, shCTRL $n=5$, shSTX $5 n=7$, $t$-test, $* * * * P<0.0001)$. Examples of continuous and fragmented Golgis stained with TGN46 (gray) are shown. (F) WPB length in shSTX5 cells with continuous versus fragmented Golgi (median $\pm I R, n=6$, t-test, $* * \star P<0.001)$. 
pletion (Online Supplementary Figure S5). These dispersed Golgi structure in shSTX5 cells produced shorter WPB, indicating that WPB length is dependent on extended Golgi ribbon organization (Figure $3 F$ ), in agreement with previous literature. ${ }^{13,16}$ Interestingly, SEC22B was no longer apparent on the dispersed Golgi structured, and instead prominent staining surrounding the nucleus was observed (Online Supplementary Figure S6). Together these results indicate that STX5 is needed for the formation of elongated WPB, by controlling the maintenance of extended Golgi stacks that allow for loading of multiple VWF quanta into newly forming WPB.

\section{No rough endoplasmic reticulum dilation due to von Willebrand factor retention upon STX5 silencing} We used transmission electron microscopy to further evaluate the morphology of WPB, Golgi, and the ER (Figure 4A). Similar as earlier noted by light microscopy, the length of the WPB (Figure 4A; WPB indicated by cyan overlay) was reduced upon STX5 silencing (Figure 4B). Golgi ribbon structures arranged in compact stacks were clearly identifiable in shCTRL cells, whereas shSTX5 cells contained widely dispersed Golgi fragments (Figure 4A; Golgi indicated by green overlay), which were a challenge to locate despite using the centriole as a reference point (Figure $4 \mathrm{~A}$; centrioles indicated by magenta overlay). As a consequence, the majority of shSTX 5 cells that were analyzed by transmission electron microscopy seemingly lacked elongated Golgi stacks entirely (Figure 4C). These observations, along with immunofluorescence microscopy images showing an altered morphology of the Golgi apparatus in STX5-deficient cells (Figure 3C, Online Supplementary Figure S5), support the emerging concept that formation of elongated WPB depends on the presence of a TGN with extended ribbons to package multiple VWF quanta in nascent WPB. ${ }^{13,14}$

We previously showed that SEC22B depletion causes retention of VWF inside the ER lumen and massive dilation of the rough ER as the SEC22B-dependent anterograde trafficking pathway to the Golgi is blocked. ${ }^{16}$ STX5 depletion also caused some retention of VWF in the ER as judged by the overlap of a perinuclear pool of VWF with
A shCTRL
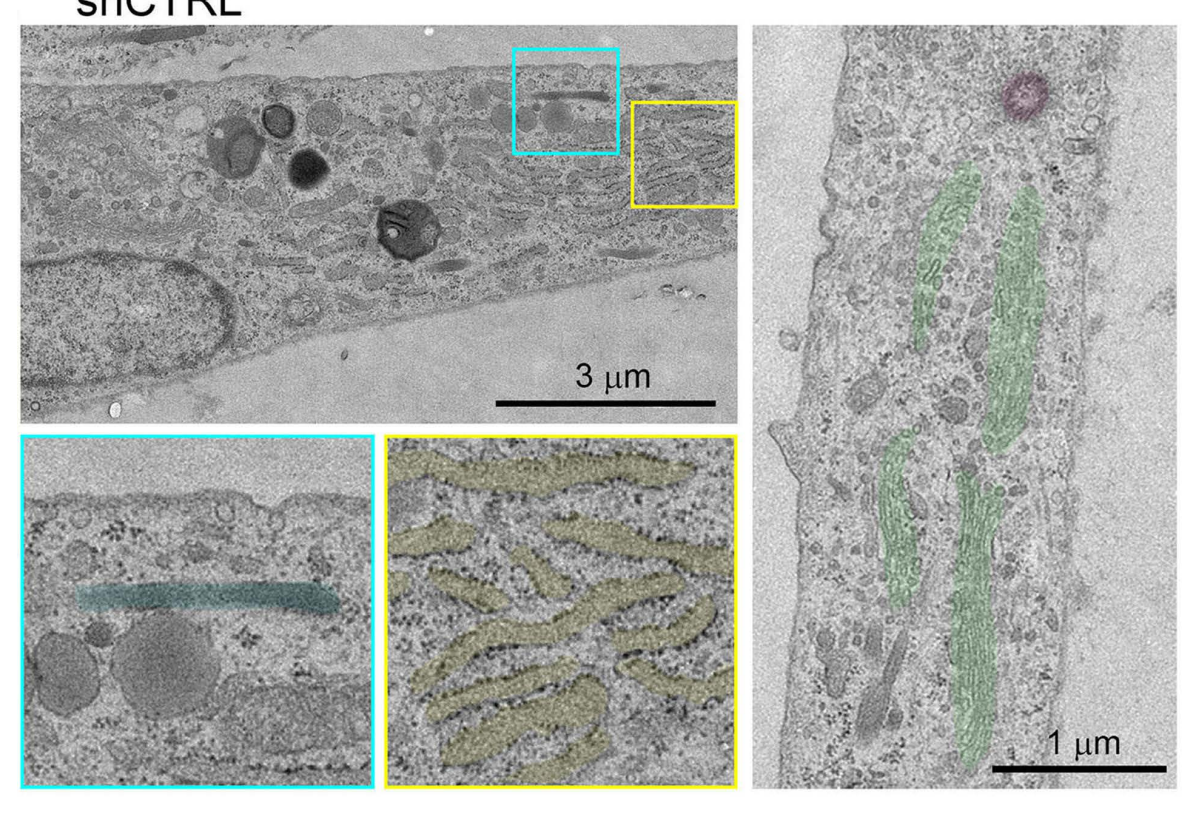

shSTX5
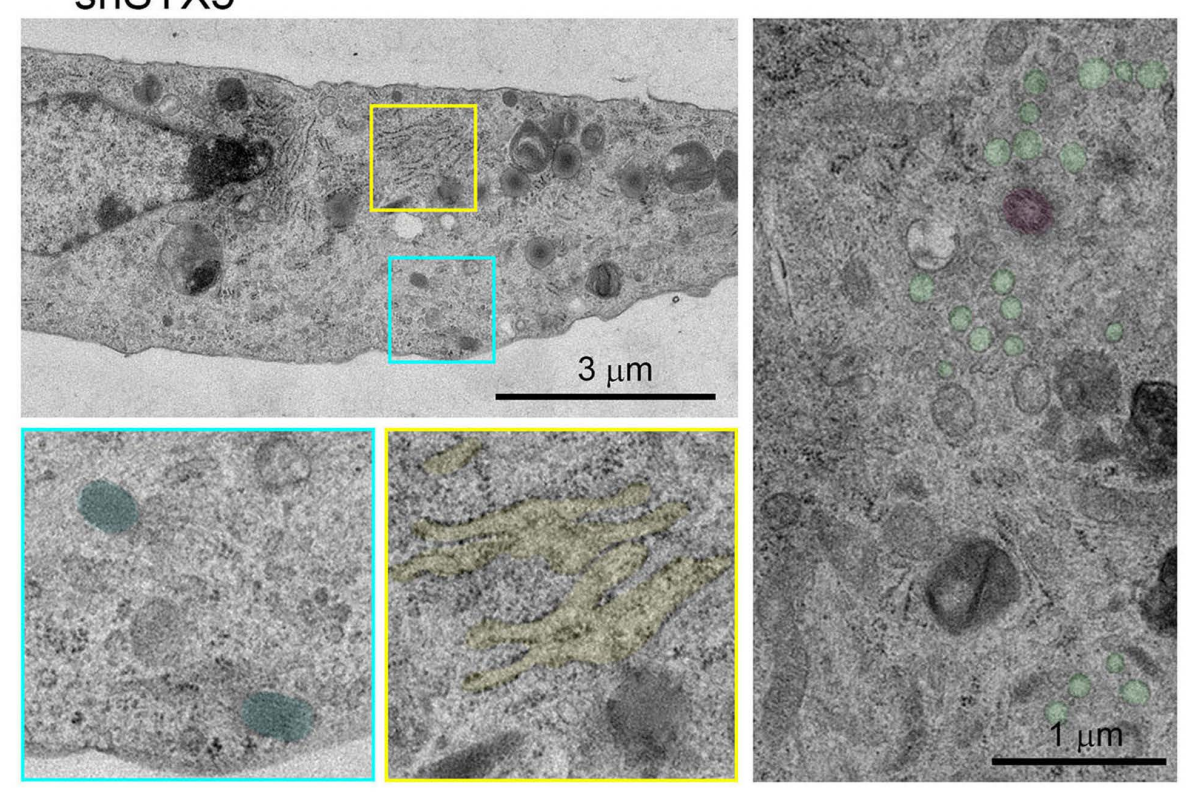

B

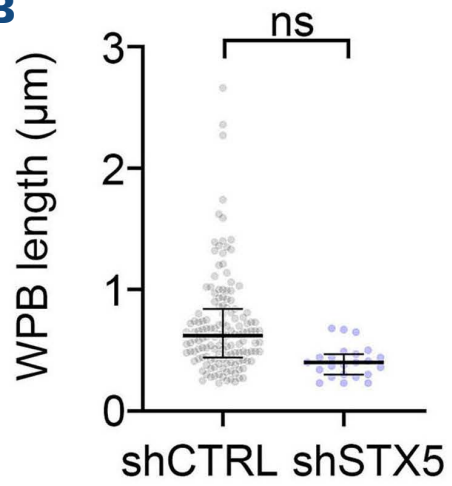

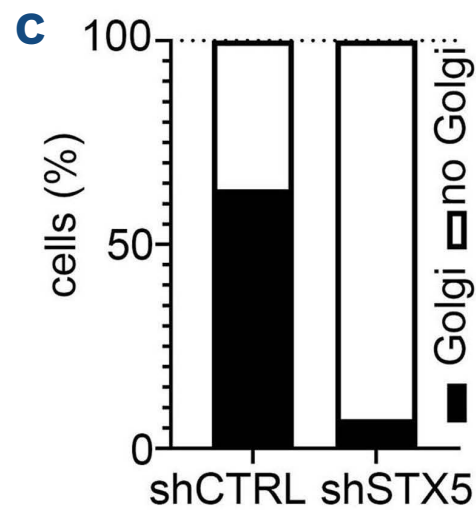

D

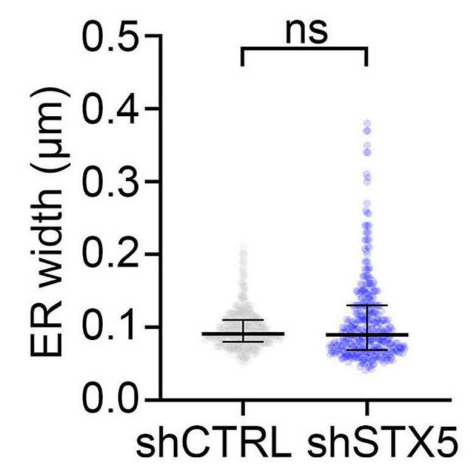

Figure 4. STX5 depletion induces morphological changes in Golgi and rough endoplasmic reticulum. (A) Transmission electron microscopy images of shCTRL and shSTX5 cells. Boxed regions are magnified below outlined with corresponding colors. WeibelPalade bodies (WPB) and rough endoplasmic reticulum (ER) sheets are highlighted with cyan and yellow overlays, respectively. In the image on the right, Golgi segments are highlighted in green and centrioles in magenta. Scale bars represent $3 \mu \mathrm{m}$ and 1 $\mu \mathrm{m}$ as indicated. (B) WPB length measured in transmission electron microscopy images (median \pm interquartile range [IR], $t$-test, $* * * P<0.001)$. (C) The percentage of cells with Golgi ribbons versus no Golgi ribbons. (D) The rough ER sheet width measured in shCTRL and shSTX5 cells (median $\pm I R, n=3$, $t$-test, $n s$ : not significant). 
the rough ER marker Protein disulfide isomerase A3 (PDI) (Online Supplementary Figure S7). Morphological analysis of the ER revealed that STX5 silencing did not cause VWF retention to the same extent as observed previously in SEC22B-depleted cells, ${ }^{16}$ as no large round dense (VWFpositive) structures were detected inside the ER lumen (Figure 4A; rough ER sheets indicated with yellow overlay). Modest dilation of the rough ER was occasionally observed upon STX5 knockdown (Online Supplementary Figure S8; rough ER sheets indicated with yellow overlay), but on average the luminal width of rough ER cisternae was not significantly altered in the shSTX5 cells (Figure 4D). Overall, these results indicate that while STX5 and SEC22B depletion have a comparable impact on WPB and Golgi morphology, the increase of ER volume to accommodate accumulating (secretory) proteins is unique to SEC22B knockdown.
Characteristic Weibel-Palade body markers co-localize with von Willebrand factor-positive structures in shSTX5 cells

Since WPB contain an array of proteins besides $\mathrm{VWF}^{8}$ we examined whether the localization of these proteins to WPB depends on STX5 by analyzing several WPB cargo and WPB membrane-associated proteins using immunofluorescence microscopy. The soluble angiogenic mediator Ang-2 and transmembrane adhesion receptor P-selectin (also referred to as CD62P) are sorted to the WPB during biogenesis at the TGN. ${ }^{36-38}$ Confocal imaging showed that Ang-2 and P-selectin both localized at WPB in ShCTRL as well as shSTX5 cells, implying that trafficking of these proteins from the Golgi to their storage compartment continues despite the loss of elongated Golgi architecture upon STX5 silencing (Figure 5A, B). We also investigated localization of two late-stage WPB markers: Rab27A and CD63, each representative of a separate route for postGolgi protein arrival to WPB. Rab27A, an established matu-
A
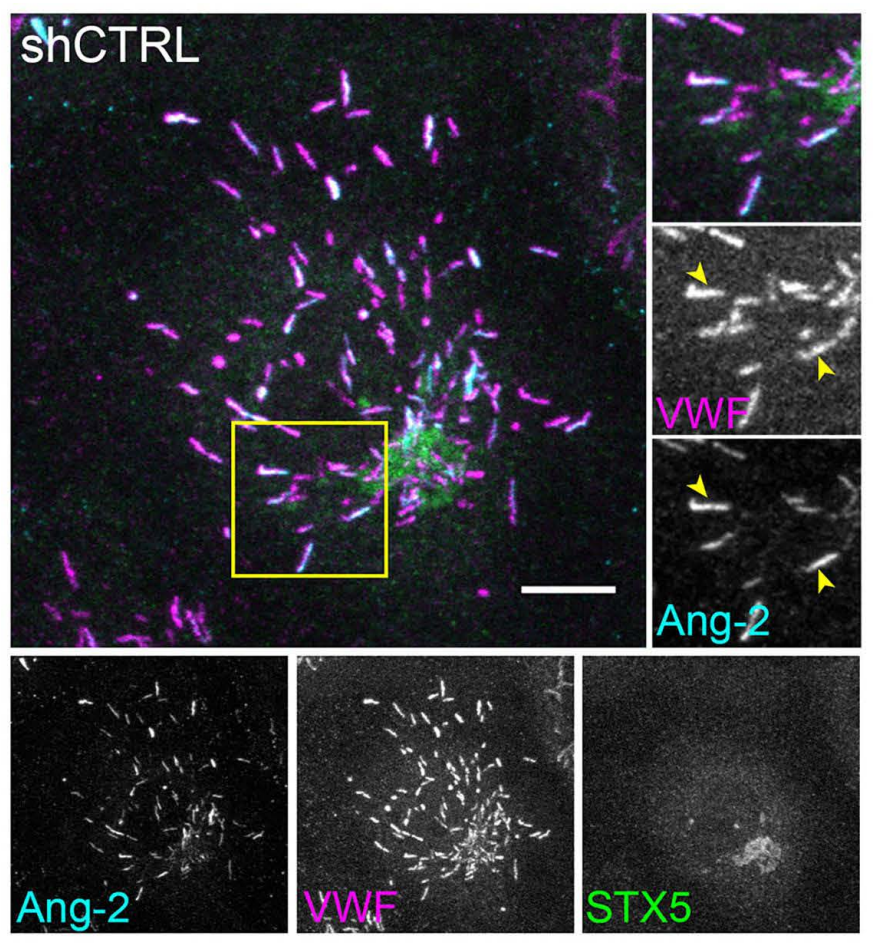

B

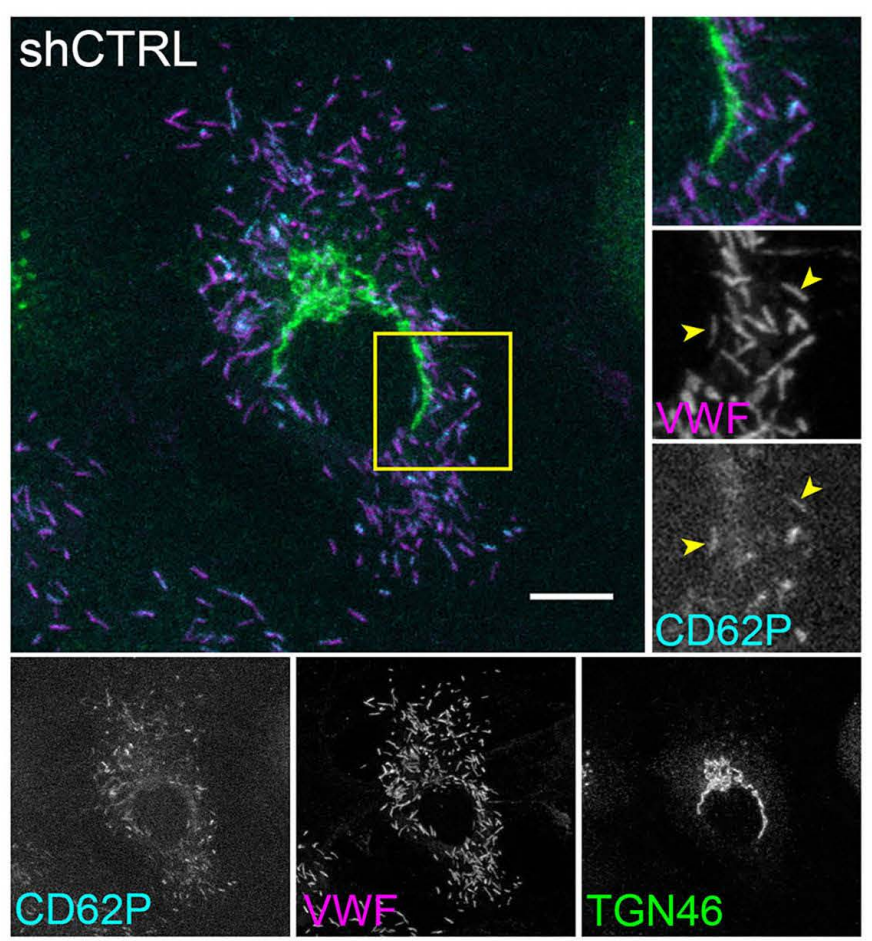

shSTX5
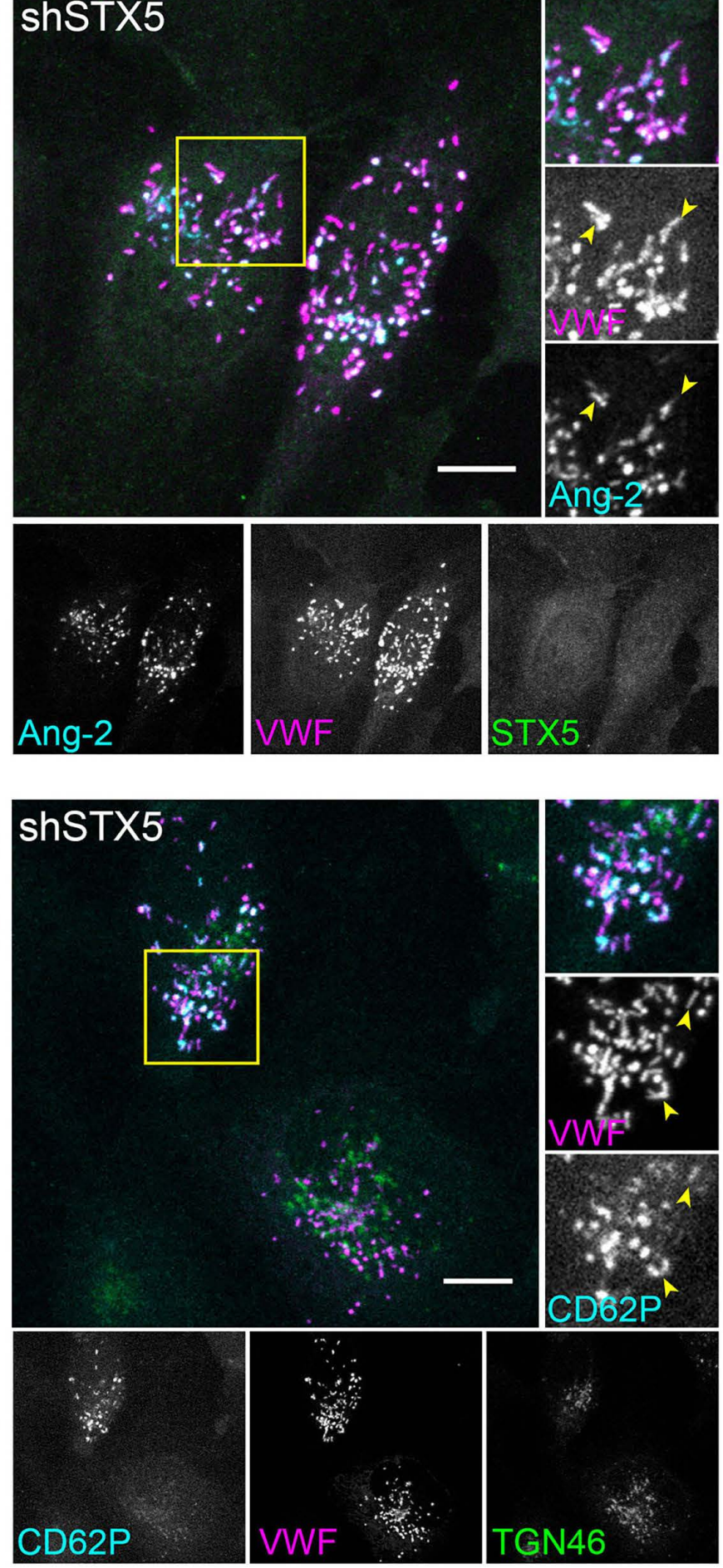

Figure 5. Weibel-Palade body cargo proteins Angiopoietin-2 and P-selectin localize to small Weibel-Palade bodies in STX5-depleted cells. (A) Immunofluorescent staining of Angiopoietin 2 (Ang2, cyan), von Willebrand factor (VWF, magenta), and STX5 (green) and (B) $\mathrm{P}$-selectin (CD62P, cyan), VWF (magenta), and TGN46 (green) in shCTRL and shSTX5 human umbilical vein endothelial cells (scale bar: $10 \mu \mathrm{m}$ ). Individual channels are shown below in gray scale. Boxed areas are magnified on the right. Yellow arrowheads indicate Ang-2-positive and $P$-selectin-positive WPB in (A) and (B), respectively. 

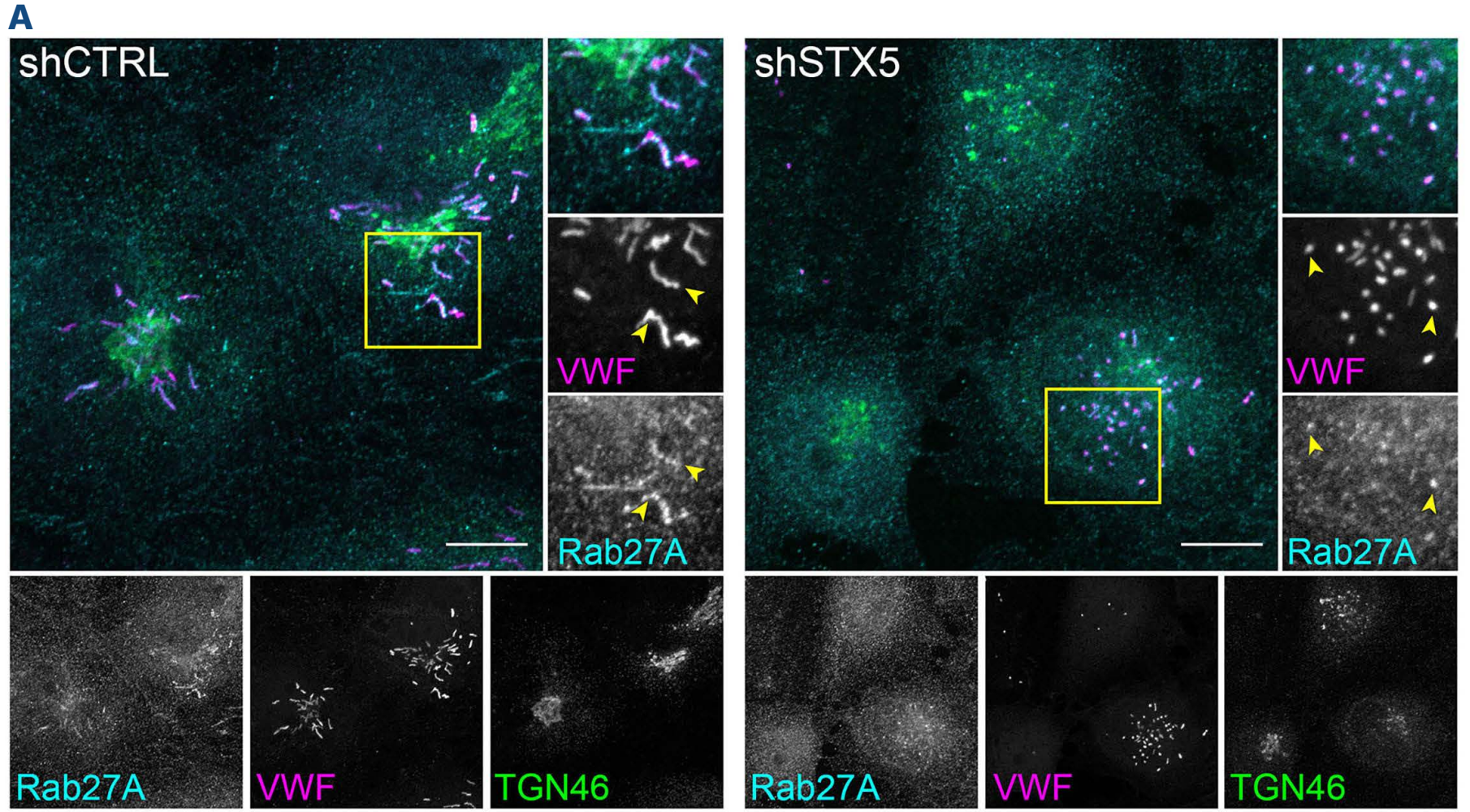

Figure 6. Normal recruitment of maturation marker Rab27A and endosome-derived CD63 to small Weibel-Palade bodies upon STX5 knockdown. (A) Immunofluorescent staining of Rab27A (cyan), VWF (magenta), and STX5 (green) and (B) CD63 (cyan), VWF (magenta), and TGN46 (green) in ShCTRL and shSTX5 human umbilical vein endothelial cells (scale bar: $10 \mu \mathrm{m}$ ). Individual channels are shown below in gray scale. Boxed areas are magnified on the right. Yellow arrowheads indicate Rab27A-positive and CD63-positive Weibel-Palade bodies in (A) and (B), respectively.

B
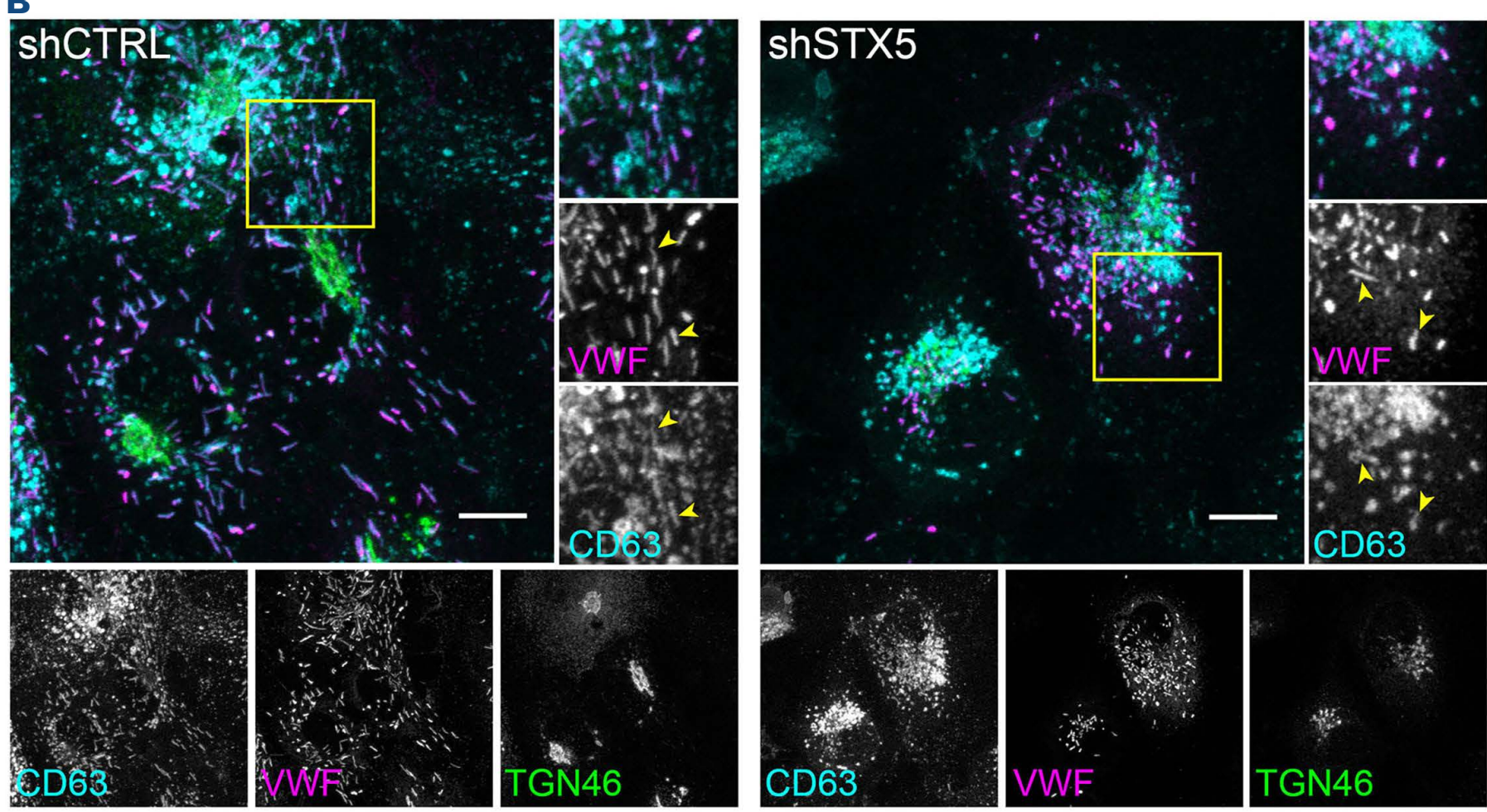

ration marker that is mobilized from the cytosol and recruits essential exocytosis machinery to the WPB membrane ${ }^{39-41}$ was present on the small WPB in STX5-depleted EC (Figure 6A). CD63, an integral membrane protein that reaches the WPB from late endosomes via an AP-3-dependent mechanism, ${ }^{20,42,43}$ was observed on spherical compartments, likely endosomes, and elongated WPB in shCTRL cells as well as rounded WPB in shSTX5 cells, indicating that its recruitment from endosomes was not impaired by STX5 depletion (Figure 6B). Thus, localization of a selection of established WPB markers remains unchanged after STX5 silencing, indicating that trafficking of cytosolic-, Golgi- and endosome-derived cargo is not impaired.
Short Weibel-Palade bodies in STX5-depleted cells are secretion-incompetent

A previous report described that WPB size is correlated to the length and adhesive properties of VWF strings. ${ }^{11}$ Since loss of STX5 expression results in shorter WPB, we hypothesized that stimulated release of these WPB results in the formation of shorter VWF strings. To test this we perfused EC under 2.5 dynes $/ \mathrm{cm}^{2}$ flow conditions with histamine to induce WPB exocytosis and a fluorescent VWF antibody to detect VWF strings. A large number of VWF strings of varying lengths were formed on the surface of shCTRL cells, but strikingly no VWF string formation was seen for shSTX5 cells (Figure 7A). Confocal analysis of unstimulated conditions revealed that small WPB were present in shSTX5 cells, predominantly localized in the perinuclear area (Online Supplementary Figure S9). However, the absence of VWF string formation indicated that 


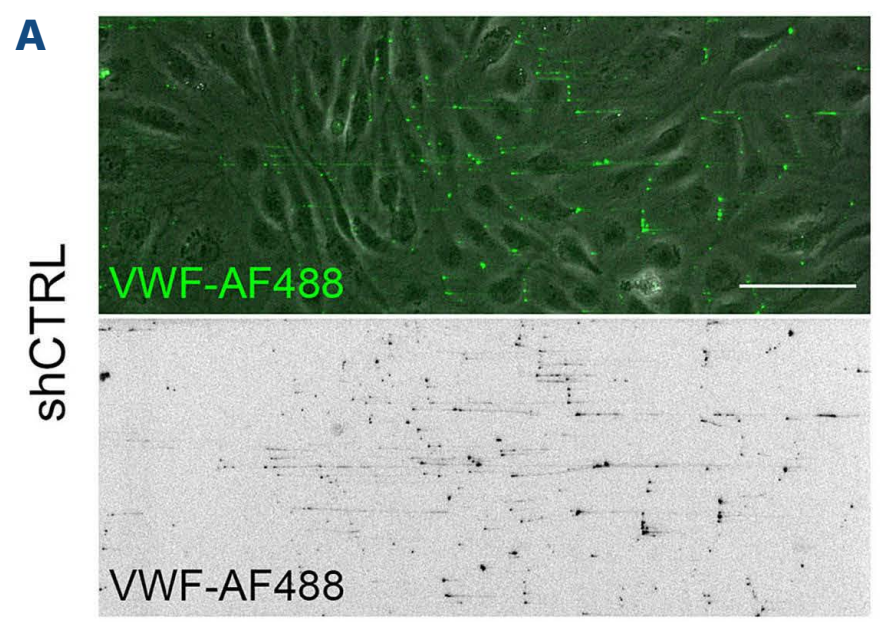

B
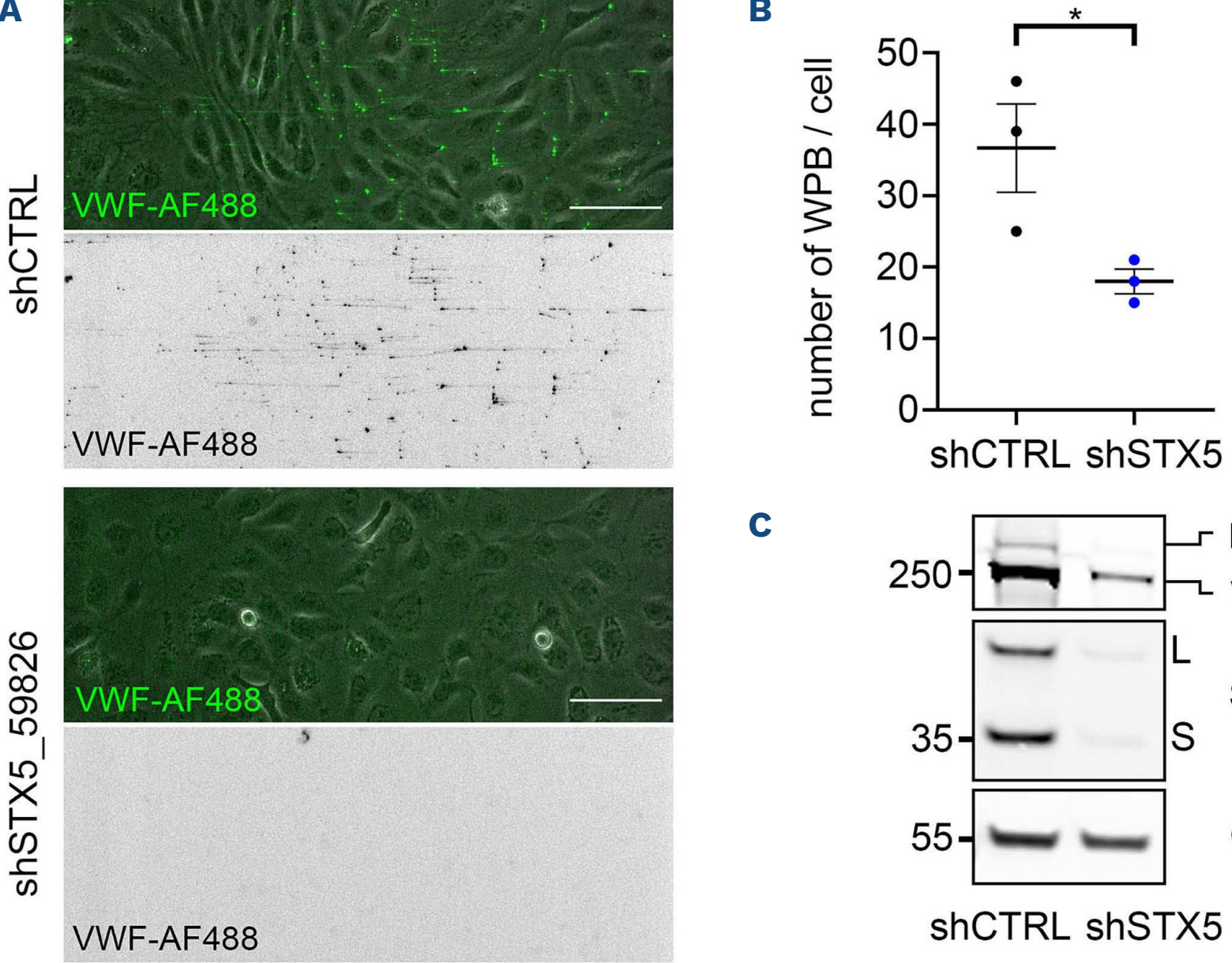

C

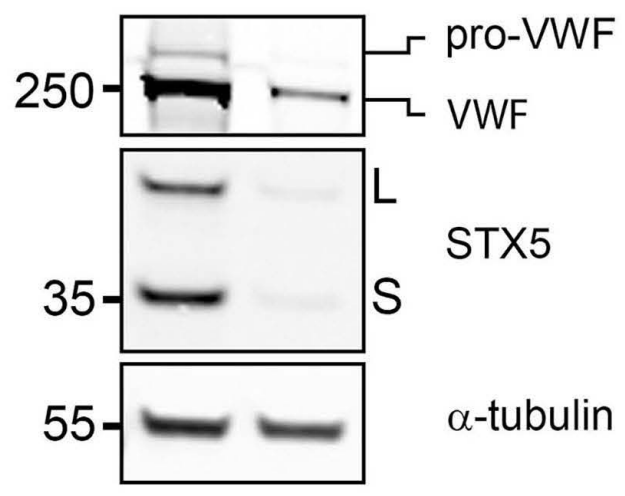

ShCTRL ShSTX5
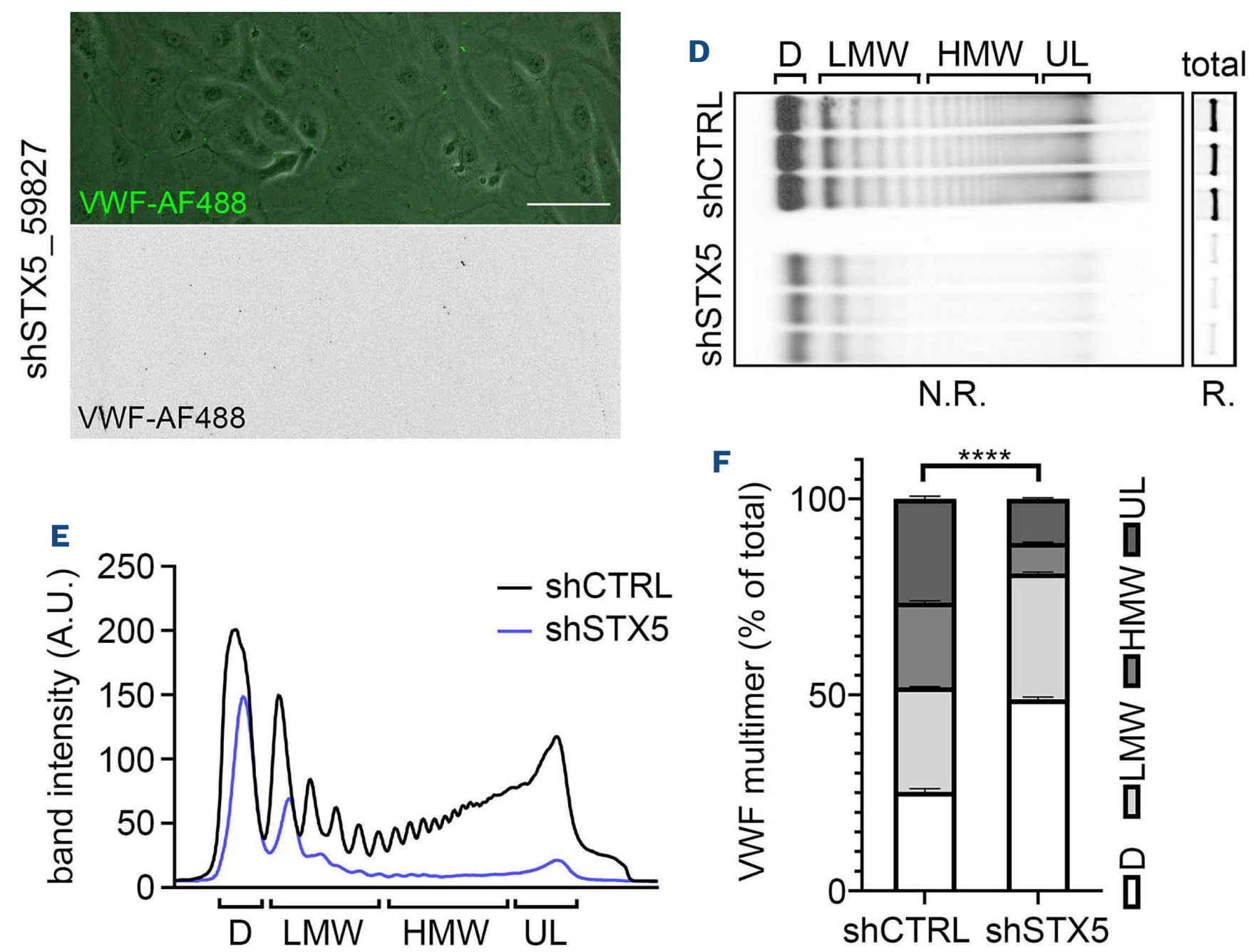

Figure 7. STX5 silencing impairs von Willebrand factor string formation. (A) von Willebrand factor (VWF) string assay with shCTRL and shSTX5-transduced human umbilical vein endothelial cells following stimulation with $100 \mu \mathrm{M}$ histamine. Extracellular VWF is shown in green in differential interference contrast and fluorescence overlay images and in black in inverted fluorescence images below (scale bar: $100 \mu \mathrm{m}$ ). (B) The average number of Weibel-Palade bodies (WPB) per cell in shCTRL and shSTX5 cells (mean \pm standard error of mean [SEM], $n=3, t$-test, $\left.{ }^{*} P<0.05\right)$. (C) Western blot analysis of VWF and STX5 expression in shCTRL and shSTX5 cells ( $\alpha$-tubulin as loading control). $L$ and $S$ indicate long and short STX5 isoforms, respectively. Molecular weights of protein marker bands are indicated in kDa. (D) VWF multimer analysis on media secreted over $24 \mathrm{~h}$ by shCTRL and shSTX5 cells. D: dimer, LMW: low molecular weight, HMW: high molecular weight, UL: ultra large. N.R.: non-reducing conditions, R.: reducing conditions. (E) Band intensity profile plot of representative VWF multimer patterns in arbitrary units (A.U.). (F) Quantification of the area under the curve (AUC) of D, LMW, HMW, and UL multimers as a percentage of total AUC per curve (mean \pm $\mathrm{SEM}, \mathrm{n}=3$, two-way analysis of variance, $* \star * * P<0.0001)$. 
these WPB are secretion-incompetent upon stimulation. Immunofluorescent analysis of WPB in shSTX5 and ShCTRL cells showed that in addition to size, the average number of WPB per cell was significantly reduced upon STX5 knockdown (Figure 7B). To examine whether the observed decrease in WPB numbers was due to downregulated VWF expression in STX5-depleted cells, we quantified intracellular VWF levels by western blot and enzyme-linked immunosorbent assay (Figure 7C, Online Supplementary Figure S10A). In line with the reduction of WPB, intracellular VWF levels were significantly reduced upon STX5 silencing, which could indicate a decreased expression, or an increased degradation or secretion. Consistent with the VWF string assay data histamine-stimulated secretion of VWF was sharply reduced following STX5 silencing, emphasizing that the small WPB in STX5 knockdown cells are stimulus-insensitive (Online Supplementary Figure $S 10 B$, $C)$. Unstimulated VWF secretion, which is a composite of basal, WPB-derived secretion and constitutive secretion, was decreased, but not proportionate to stored VWF (Online Supplementary Figure S10D, E). Subsequent VWF multimer analysis of the unstimulated releasates from shCTRL and shSTX5 cells revealed a remarkable change in multimer size composition, with relatively more VWF dimers and low molecular weight multimers, but less high molecular weight and ultra-large multimers secreted by STX5-depleted cells (Figure 7D-F). Notably, shSTX5-derived VWF dimers and low molecular weight multimers showed a slightly reduced mobility in gel electrophoresis, possibly indicating differences in post-translational modification.

Collectively, these data indicate that loss of STX5 has critical consequences for WPB biogenesis, VWF string formation, secretion, and multimerization, indicating that this SNARE protein is a crucial player in the endothelial secretory pathway.

\section{Discussion}

SNARE proteins constitute the key machinery that promotes membrane fusion during vesicle transport. ${ }^{19}$ We have previously identified several SNARE proteins involved in WPB maturation and exocytosis, while recently we have shown the critical role of SEC22B, an ER-to-Golgi SNARE, in VWF trafficking and WPB formation. . $^{16,21}$ In this study, a mass spectrometry-based approach elucidated the SEC22B interactome in EC and identified potential novel regulators of WPB biogenesis. We focused on STX5, a cognate SNARE protein that is primarily found in the Golgi membrane and has been shown to facilitate ER-to-Golgi and intra-Golgi protein trafficking. ${ }^{29}$ By silencing STX5 expression, we discovered a severe defect in WPB biogenesis, accompanied by fragmentation of the Golgi and abrogation of secretagogue-induced VWF release. Our data point to a crucial role for STX5-containing SNARE complexes in the ability of EC to efficiently store and secrete VWF.

The importance of VWF transport in WPB biogenesis is underlined by previous studies showing that WPB size depends on anterograde VWF delivery and Golgi morphology, and ultimately determines their functionality in hemostasis. ${ }^{11-13,44}$ Recently, novel players have been identified that modulate biosynthetic pathways crucially involved in WPB biogenesis. ${ }^{15,16}$ The Arf GTPases Arf1 and Arf4 and their GEF GBF1 are essential for the formation of WPB through regulation of membrane fission. ${ }^{15}$ GBF1 deficiency was accompanied by the formation of giant, secretion-incompetent WPB. Contrarily, v-SNARE SEC22B regulates ER-to-Golgi transport by facilitating vesicle fusion and depletion of SEC22B in EC blocks anterograde VWF transport and results in fragmented Golgi structures and shorter WPB. ${ }^{16}$ To this we can now add STX5, the depletion of which shows considerable phenotypic overlap with that of SEC22B-deficient cells. These studies indicate that regulators of vesicle fission and fusion are essential components of the trafficking pathways utilized for VWF multimer assembly as well as WPB biogenesis. Interestingly, GBF1 was also a hit in our SEC22B interactome screen, suggesting that there are opportunities for crosstalk between these two pathways.

STX5 is an integral member of the Golgi apparatus and can form complexes with SEC22B, which is localized on ER-derived vesicles, facilitating their fusion with the Golgi (Figure 8). ${ }^{18,29}$ While STX5L localizes predominantly to the ER, STX5S has been shown to be crucial for intra-Golgi traffic, but not Golgi morphology. ${ }^{45}$ We observed that STX5 was mainly present in the Golgi, whereas SEC22B exhibited a broader localization. Knockdown of both STX5 isoforms caused fragmentation of Golgi stacks into dispersed mini-stacks, similar to the effect of SEC22B depletion. ${ }^{16}$ Therefore, we hypothesize that Golgi fragmentation is caused by the reduced delivery of membrane or structural proteins that maintain Golgi architecture from anterograde ER-derived vesicle transport. Alternatively, as STX5 has been reported to participate in Golgi reassembly after mitosis, ${ }^{46}$ reduced STX5 levels in EC could potentially interfere with the post-mitotic Golgi restoration, leading to permanent Golgi dispersal.

Golgi architecture has a large impact on WPB biogenesis and elongation. According to the model first proposed by Ferraro et al., ${ }^{16}$ continuous, extended Golgi ribbons allow for the integration of more VWF quanta, resulting in elongated WPB, while Golgi ministacks give rise to shorter WPB. ${ }^{13}$ In keeping with the VWF quanta model, WPB length is significantly reduced in STX5-depleted cells with dispersed Golgi (Figure 8).

VWF release can be separated into three modes: consti- 


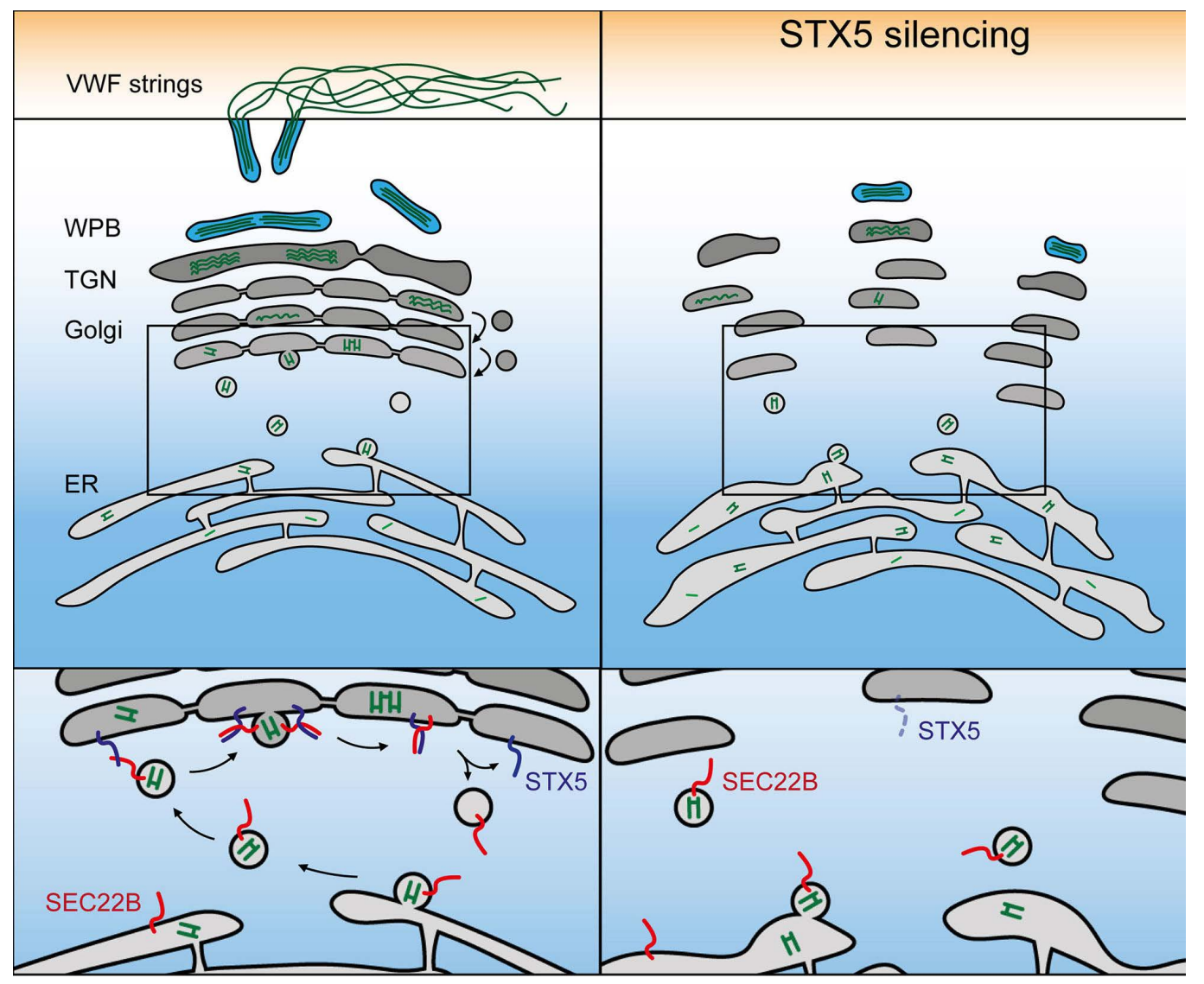

Figure 8. Proposed role of STX5 in the endothelial secretory pathway. Schematic overview of the endothelial secretory pathway (top left panel) showing von Willebrand factor (VWF, green) monomers form dimers in the endoplasmic reticulum (ER); the dimers are transported in vesicles to the Golgi (boxed area). The lower left panel depicts the proposed roles of SEC22B (red) and STX5 (blue) in this process. SEC22B, which is incorporated into the membrane of a vesicle budding off from the ER, forms a SNARE complex with STX5 present on the Golgi, facilitating membrane fusing and releasing the cargo (i.e., VWF dimers) into the Golgi lumen. Once arrived at the Golgi, VWF dimers multimerize and form quanta that arrive in the trans-Golgi network (TGN) to be packaged into a Weibel-Palade body (WPB). The WPB are transported to the plasma membrane where they can be secreted. Upon stimulation the WPB undergoes regulated exocytosis and releases its content into the vasculature, while VWF multimers form long strings. If STX5 is silenced (top right panel), more VWF is observed in the ER and the entire Golgi apparatus becomes fragmented and dispersed. Upon STX5 depletion, ER-derived vesicles can no longer fuse efficiently with the Golgi membrane (lower right panel) and only a limited amount of VWF reaches the TGN. WPB size is strongly reduced and regulated VWF secretion is diminished due to inefficient VWF anterograde transport and fragmented TGN morphology.

tutive, basal and regulated (stimulus-induced) VWF secretion. ${ }^{3}$ The WPB compartment ensures a rapid delivery of VWF to form strings and initiate hemostasis. ${ }^{7}$ Interestingly, histamine-stimulated VWF secretion was severely affected in STX5-depleted cells, as evidenced by the (nearly) absent string formation and secretion. This could be explained by the strongly depleted stimulus-sensitive compartment, i.e., reduced number of peripheral WPB, decrease in WPB length and reduced overall VWF expression levels. Recently, it has been shown that EC control functional responses by size selection of WPB during exocytosis, with a preference for longer WPB to undergo exocytosis following stimulation with a subset of secretagogues, in order to control functional responses. ${ }^{44}$ Thus, the relative unresponsiveness to histamine could also be a consequence of the reduced length of WPB in ShSTX5 cells.

Unstimulated VWF release was also decreased upon STX5 knockdown; however, the amount of secreted VWF relative to stored VWF was proportionally increased compared to the control, suggesting that basal secretion continues and is even slightly increased. Moreover, the secreted VWF contained fewer larger multimers, indicating a multimerization defect. The observed shift in size of VWF dimers could perhaps be explained by incomplete glycosylation of VWF as a result of mislocalized glycosylation enzymes that normally reside in the different Golgi stacks of the Golgi. ${ }^{47}$ Therefore, we can hypothesize that an essential role of STX5 is to maintain a continuous Golgi from which properly multimerized VWF with post-translational modifications can be stored in elongated organelles, which have the capacity to mature into a stimulus-sensitive WPB.

Partial quantitative VWF deficiency, known as VWD type 1 (VWD1), is the most prevalent subtype of VWD. ${ }^{2} A$ sizeable number of pathogenic VWF mutations in VWD1 lead to reduced plasma levels due to retention within EC, ${ }^{48-50}$ which is often accompanied by lower WPB numbers and loss of their characteristic elongated morphology. ${ }^{51,52}$ In about $25 \%$ of VWD1 patients and about $60 \%$ of individuals with "low VWF" no causal mutation can be detected in their $V W F$ gene. It has been postulated that the genetic regulation of VWF levels largely depends on an interplay of several quantitative trait loci that influence VWF synthesis 
and clearance. ${ }^{53}$ While a large portion of the heritable variation in VWF is still unaccounted for, some of these modifiers include SNARE proteins that can influence WPB exocytosis. ${ }^{25,26,53,54}$ Given that a substantial portion of quantitative VWF deficiencies in the group with VWF mutations are characterized by defects in intracellular trafficking of VWF, it is plausible that VWD and low VWF pathogenesis in cases without damaging VWF mutations may also be driven by genetic variations in cellular components that control correct progression of VWF through the endothelial secretory pathway. The SEC22B interactome determined in this study uncovered an extensive network consisting of SNARE and their regulators and components of a number of vesicle tethering complexes that operate at the ER (NRZ complex), Golgi (COG complex) and post-Golgi (exocyst). Further cellular studies are needed to elucidate their importance in VWF trafficking. Potentially, data from such "trafficome" studies can be used to inform genetic and genomic studies that focus on identifying modifiers of VWF plasma levels in patients who suffer from bleeding or thrombosis.

\section{Disclosures}

No conflicts of interest to disclose.

\section{Contributions}

$M K, E K, A J H, P E B, A A M, F P J V A$, and JO performed research and analyzed data; $D G$ provided vital reagents; $M v d B$ provided vital expertise; $M K, E K, C M, J V$, and RB designed the research and wrote the paper.

\section{Acknowledgments}

We would like to thank Dr. R.I. Koning and Dr. C.R. Jost for their critical reading of the manuscript.

\section{Funding}

Work in our laboratory was funded by grants from the Landsteiner Stichting voor Bloedtransfusie Research (LSBR-1707) and the Dutch Thrombosis Foundation (TSN 2017-01).

\section{Data-sharing statement}

The mass spectrometry proteomics data, including the .raw mass spectrometry files and search/identification files obtained with MaxQuant have been deposited with the ProteomeXchange Consortium (http://proteomecentral.proteomexchange.org) via the PRIDE partner repository with the dataset identifier PXD027516.

\section{References}

1. Sadler JE. von Willebrand factor assembly and secretion. J Thromb Haemost. 2009;7(Suppl 1):24-27.

2. Leebeek FWG, Eikenboom JCJ. von Willebrand's disease. N Engl J Med. 2016;375(21):2067-2080.

3. Lopes da Silva M, Cutler DF. von Willebrand factor multimerization and the polarity of secretory pathways in endothelial cells. Blood. 2016;128(2):277-285.

4. Wagner DD, Marder VJ. Biosynthesis of von Willebrand protein by human endothelial cells. J Biol Chem. 1983;258(4):2065-2067.

5. Voorberg J, Fontijn R, Calafat J, Janssen $\mathrm{H}$, van Mourik JA, Pannekoek $\mathrm{H}$. Biogenesis of von Willebrand factor-containing organelles in heterologous transfected CV-1 cells. EMBO J. 1993;12(2):749-758.

6. Schillemans M, Kat M, Westeneng J, et al. Alternative trafficking of Weibel-Palade body proteins in CRISPR/Cas9-engineered von Willebrand factor-deficient blood outgrowth endothelial cells. Res Pract Thromb Haemost. 2019;3(4):718-732.

7. Schillemans M, Karampini E, Kat M, Bierings R. Exocytosis of Weibel-Palade bodies: how to unpack a vascular emergency kit. J Thromb Haemost. 2019;17(1):6-18.

8. McCormack JJ, Lopes da Silva M, Ferraro F, Patella F, Cutler DF. Weibel-Palade bodies at a glance. J Cell Sci.

2017;130(21):3611-3617.

9. Valentijn KM, Valentijn JA, Jansen KA, Koster AJ. A new look at Weibel-Palade body structure in endothelial cells using electron tomography. J Struct Biol. 2008;161(3):447-458.

10. Berriman JA, Li S, Hewlett LJ, et al. Structural organization of Weibel-Palade bodies revealed by cryo-EM of vitrified endothelial cells. Proc Natl Acad Sci U S A. 2009;106(41):17407-17412.
11. Ferraro F, Lopes-da-Silva M, Grimes W, et al. Weibel-Palade body size modulates the adhesive activity of its von Willebrand factor cargo in cultured endothelial cells. Sci Rep. 2016;6(1):32473.

12. Nightingale TD, McCormack JJ, Grimes W, et al. Tuning the endothelial response: differential release of exocytic cargos from Weibel-Palade bodies. J Thromb Haemost. 2018;16(9):1873-1886.

13. Ferraro F, Kriston-Vizi J, Metcalf DJ, et al. A two-tier Golgibased control of organelle size underpins the functional plasticity of endothelial cells. Dev Cell. 2014;29(3):292-304.

14. Mourik MJ, Faas FGA, Zimmermann H, Voorberg J, Koster AJ, Eikenboom J. Content delivery to newly forming Weibel-Palade bodies is facilitated by multiple connections with the Golgi apparatus. Blood. 2015;125(22):3509-3516.

15. Lopes-da-Silva M, McCormack JJ, Burden JJ, Harrison-Lavoie KJ, Ferraro F, Cutler DF. A GBF1-dependent mechanism for environmentally responsive regulation of ER-Golgi transport. Dev Cell. 2019;49(5):786-801.

16. Karampini E, Bürgisser PE, Olins J, et al. Sec22b determines Weibel-Palade body length by controlling anterograde ER-Golgi transport. Haematologica. 2021;106(4):1138-1147.

17. Karampini E, Bierings R, Voorberg J. Orchestration of primary hemostasis by platelet and endothelial lysosome-related organelles. Arterioscler Thromb Vasc Biol. 2020;40(6):1441-1453.

18. Brandizzi F, Barlowe C. Organization of the ER-Golgi interface for membrane traffic control. Nat Rev Mol Cell Biol. 2013;14(6):382-392.

19. Jahn R, Scheller RH. SNAREs--engines for membrane fusion. Nat Rev Mol Cell Biol. 2006;7(9):631-643. 
20. Karampini E, Schillemans M, Hofman M, et al. Defective AP-3dependent VAMP8 trafficking impairs Weibel-Palade body exocytosis in Hermansky-Pudlak syndrome type 2 blood outgrowth endothelial cells. Haematologica. 2019;104(10):2091-2099.

21. Schillemans M, Karampini E, van den Eshof BL, et al. WeibelPalade body localized Syntaxin-3 modulates von Willebrand factor secretion from endothelial cells. Arterioscler Thromb Vasc Biol. 2018;38(7):1549-1561.

22. Zhu Q, Yamakuchi M, Lowenstein CJ. SNAP23 regulates endothelial exocytosis of von Willebrand factor. PLoS One. 2015;10(8):e0118737.

23. Pulido IR, Jahn R, Gerke V. VAMP3 is associated with endothelial Weibel-Palade bodies and participates in their $\mathrm{Ca}(2+)$ dependent exocytosis. Biochim Biophys Acta. 2011;1813(5):1038-1044.

24. van Breevoort D, Snijders AP, Hellen N, et al. STXBP1 promotes Weibel-Palade body exocytosis through its interaction with the Rab27A effector Slp4-a. Blood. 2014;123(20):3185-3194.

25. van Loon JE, Sanders YV, de Wee EM, Kruip MJ, de Maat MP, Leebeek FW. Effect of genetic variation in STXBP5 and STX2 on von Willebrand factor and bleeding phenotype in type 1 von Willebrand disease patients. PLoS One. 2012;7(7):e40624.

26. Van Loon J, Dehghan A, Weihong T, et al. Genome-wide association studies identify genetic loci for low von Willebrand factor levels. Eur J Hum Genet. 2016;24(7):1035-1040.

27. Schillemans M, Karampini E, Hoogendijk AJ, et al. Interaction networks of Weibel-Palade body regulators syntaxin-3 and syntaxin binding protein 5 in endothelial cells. J Proteomics. 2019;205:103417.

28. Ercig B, Graça NAG, Kangro K, et al. N-glycan-mediated shielding of ADAMTS13 prevents binding of pathogenic autoantibodies in immune-mediated TTP. Blood. 2021;137(19):2694-2698.

29. Linders PTA, van der Horst C, Ter Beest M, van den Bogaart G. Stx5-mediated ER-Golgi transport in mammals and yeast. Cells. 2019;8(8):780.

30. Tagaya M, Arasaki K, Inoue H, Kimura $\mathrm{H}$. Moonlighting functions of the NRZ (mammalian Dsl1) complex. Front Cell Dev Biol. 2014;2:25.

31. Willett R, Kudlyk T, Pokrovskaya I, et al. COG complexes form spatial landmarks for distinct SNARE complexes. Nat Commun. 2013;4(1):1553.

32. Kudlyk T, Willett R, Pokrovskaya ID, Lupashin V. COG6 interacts with a subset of the Golgi SNAREs and is Important for the Golgi complex integrity. Traffic. 2013;14(2):194-204.

33. Hui N, Nakamura N, Sönnichsen B, Shima DT, Nilsson T, Warren G. An isoform of the Golgi t-SNARE, syntaxin 5, with an endoplasmic reticulum retrieval signal. Mol Biol Cel.l 1997;8(9):1777-1787.

34. Mancias JD, Goldberg J. The transport signal on Sec22 for packaging into COPII-coated vesicles is a conformational epitope. Mol Cell. 2007;26(3):403-414.

35. Knop M, Aareskjold E, Bode G, Gerke V. Rab3D and annexin A2 play a role in regulated secretion of $V W F$, but not TPA, from endothelial cells. EMBO J. 2004;23(15):2982-2992.

36. Fiedler U, Scharpfenecker M, Koidl S, et al. The Tie-2 ligand Angiopoietin-2 is stored in and rapidly released upon stimulation from endothelial cell Weibel-Palade bodies. Blood. 2004;103(11):4150-4156.

37. Bonfanti R, Furie BC, Furie B, Wagner DD. PADGEM (GMP140) is a component of Weibel-Palade bodies of human endothelial cells.
Blood. 1989;73(5):1109-1112.

38. McEver RP, Beckstead JH, Moore KL, Marshall-Carlson L, Bainton DF. GMP-140, a platelet alpha-granule membrane protein, is also synthesized by vascular endothelial cells and is localized in Weibel-Palade bodies. J Clin Invest. 1989;84(1):92-99.

39. van Breevoort D, van Agtmaal EL, Dragt BS, et al. Proteomic screen identifies IGFBP7 as a novel component of endothelial cell-specific Weibel-Palade bodies. J Proteome. Res 2012;11(5):2925-2936.

40. Hannah MJ, Hume AN, Arribas M, et al. Weibel-Palade bodies recruit Rab27 by a content-driven, maturation-dependent mechanism that is independent of cell type. $J$ Cell Sci. 2003;116(Pt 19):3939-3948.

41. Kat M, Bürgisser PE, Janssen $H$, et al. GDP/GTP exchange factor MADD drives activation and recruitment of secretory Rab GTPases to Weibel-Palade bodies. Blood Adv. 2021;5(23):5116-5127.

42. Kobayashi T, Vischer UM, Rosnoblet $C$, et al. The tetraspanin CD63/lamp3 cycles between endocytic and secretory compartments in human endothelial cells. Mol Biol Cell. 2000;11(5):1829-1843.

43. Vischer U, Wagner D. CD63 is a component of Weibel-Palade bodies of human endothelial cells. Blood. 1993;82(4):1184-1191.

44. McCormack JJ, Harrison-Lavoie KJ, Cutler DF. Human endothelial cells size-select their secretory granules for exocytosis to modulate their functional output. J Thromb Haemost. 2020;18(1):243-254.

45. Linders PTA, Gerretsen ECF, Ashikov A, et al. Congenital disorder of glycosylation caused by starting site-specific variant in syntaxin-5. Nat Commun. 2021;12(1):6227.

46. Rabouille C, Kondo H, Newman R, Hui N, Freemont P, Warren G. Syntaxin 5 is a common component of the NSF- and p97mediated reassembly pathways of Golgi cisternae from mitotic golgi fragments in vitro. Cell. 1998;92(5):603-610.

47. Linders PTA, Peters E, Ter Beest M, Lefeber DJ, van den Bogaart G. Sugary logistics gone wrong: membrane trafficking and congenital disorders of glycosylation. Int $\mathrm{J}$ Mol Sci. 2020;21(13):4654.

48. Goodeve A. Diagnosing von Willebrand disease: genetic analysis. Hematol Am Soc Hematol Educ Progr. 2016;2016(1):678-682.

49. de Jong A, Eikenboom J. Von Willebrand disease mutation spectrum and associated mutation mechanisms. Thromb Res. 2017;159:65-75.

50. Eikenboom J, Hilbert L, Ribba AS, et al. Expression of 14 von Willebrand factor mutations identified in patients with type 1 von Willebrand disease from the MCMDM-1VWD study. J Thromb Haemost. 2009;7(8):1304-1312.

51. Wang J-W, Bouwens EAM, Pintao MC, et al. Analysis of the storage and secretion of von Willebrand factor in blood outgrowth endothelial cells derived from patients with von Willebrand disease. Blood. 2013;121(14):2762-2772.

52. Starke RD, Paschalaki KE, Dyer CEF, et al. Cellular and molecular basis of von Willebrand disease: studies on blood outgrowth endothelial cells. Blood. 2013;121(14):2773-2784

53. Swystun LL, Lillicrap D. Genetic regulation of plasma von Willebrand factor levels in health and disease. J Thromb Haemost. 2018;16(12):2375-2390.

54. Smith NL, Chen M-H, Dehghan A, et al. Novel associations of multiple genetic loci with plasma levels of factor VII, factor VIII, and von Willebrand factor. Circulation. 2010;121(12):1382-1392. 